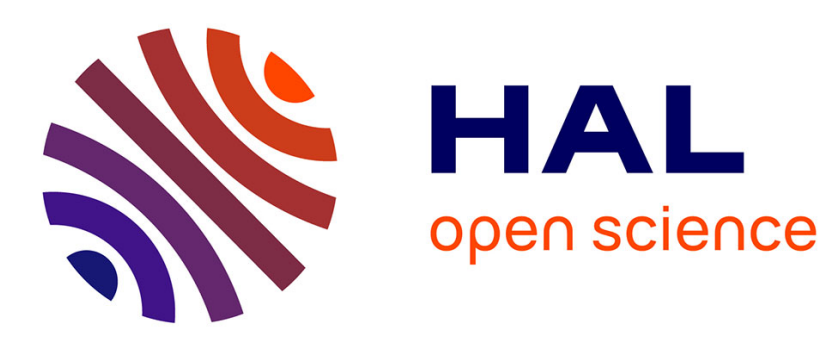

\title{
Grey-box identification of a non-linear solar array structure using cubic splines
}

Jean-Philippe Noel, G Kerschen, Emmanuel Foltête, Scott Cogan

\section{To cite this version:}

Jean-Philippe Noel, G Kerschen, Emmanuel Foltête, Scott Cogan. Grey-box identification of a nonlinear solar array structure using cubic splines. International Journal of Non-Linear Mechanics, 2014, 67, pp.106 - 119. hal-03221996

\section{HAL Id: hal-03221996 https://hal.science/hal-03221996}

Submitted on 10 May 2021

HAL is a multi-disciplinary open access archive for the deposit and dissemination of scientific research documents, whether they are published or not. The documents may come from teaching and research institutions in France or abroad, or from public or private research centers.
L'archive ouverte pluridisciplinaire HAL, est destinée au dépôt et à la diffusion de documents scientifiques de niveau recherche, publiés ou non, émanant des établissements d'enseignement et de recherche français ou étrangers, des laboratoires publics ou privés. 


\title{
Grey-box Identification of a Nonlinear Solar Array Structure using Cubic Splines
}

\author{
J.P. Noël ${ }^{1}$, G. Kerschen ${ }^{1}$, E. Foltête ${ }^{2}$, S. Cogan ${ }^{2}$ \\ ${ }^{1}$ Space Structures and Systems Laboratory \\ Department of Aerospace and Mechanical Engineering \\ University of Liège \\ Chemin des chevreuils 1, 4000, Liège, Belgium \\ ${ }^{2}$ FEMTO-ST Institute \\ Department of Applied Mechanics \\ Rue de l'épitaphe 24, 25000, Besançon, France \\ Corresponding author: Jean-Philippe Noël \\ Email: jp.noel@ulg.ac.be, phone: +32 43664854.
}

\begin{abstract}
Most identification methods in nonlinear structural dynamics assume in advance a mathematical model of the nonlinearities. This is however possible in specific situations only, since nonlinear effects may be caused by numerous phenomena and a priori knowledge is generally limited. The present paper investigates the usefulness of piecewise third-order polynomials, termed cubic splines, to identify the complex nonlinear dynamics of solar arrays in their stowed configuration. The estimation of the model parameters is achieved using the frequency-domain nonlinear subspace identification (FNSI) method. A distinct advantage of the FNSI approach is its capability to calculate accurately a large number of parameters, while maintaining an acceptable computational burden. This makes tractable the use of cubic splines to represent nonlinearity in real-life mechanical systems, as the dimensionality of the inverse problem is known to increase dramatically in this case. The experimental structure of interest consists of two parallel aluminium plates assembled with bolted connections. This application is challenging because of the existence of impacts between the two plates at high excitation amplitude, and of the activation of complicated stiffness and damping mechanisms within the bolted connections.
\end{abstract}

Keywords: Nonlinear system identification; cubic splines; frequency-domain subspace method; solar array structure; unilateral impacts; bolted connections. 


\section{Introduction}

The frequent occurrence of nonlinear behaviour in structural dynamic testing is now commonly attested, at least in certain regimes of motion. In particular, the last decade witnessed numerous works reporting evidence of nonlinearity in aerospace structures $[1$, $2,3,4]$. It has therefore become obvious that nonlinear components should be dealt with in the design process in order to satisfy the continual interest in expanding the performance envelope of engineering systems. This is why the development of effective system identification techniques applicable to nonlinear systems is today an active research area in the structural dynamics community.

Once nonlinear behaviour is detected, system identification is usually accomplished in two steps [5], namely selecting a suitable nonlinearity model based on available prior knowledge, and processing measured data to estimate model parameters. Different types of models can be distinguished by the amount of prior knowledge they incorporate, specifically white-box, grey-box and black-box models [6]. In white-box identification, the overall model structure and the mathematical representation of the nonlinearities are specified in advance based on physics. Assuming the functional form of the nonlinearities is however possible in specific situations only, as in the case of large displacement motions [7]. This is otherwise a hard requirement to handle, since nonlinear effects may be caused by numerous phenomena and a priori knowledge is generally limited. One may alternatively turn to black-box approaches, where a sufficiently rich and flexible model structure is employed $[8,9,10]$ to capture all relevant physics [11]. The major drawback of black-box models is that they make no use of the physical insights the user may possess into the system under test.

Grey-box modelling is therefore a relevant alternative. In grey-box identification, a known model structure, usually dictated by Newton's law of dynamics, is complemented with mathematical functionals that may represent a vast variety of nonlinear behaviours. The most standard practice is to resort to high-order polynomial expansions, such as ordinary [12] or Chebyshev polynomials [13]. Some authors utilised neural networks and learning algorithms to approximate nonlinearities and estimate model parameters, respectively $[14,15]$. Another approach for handling unknown nonlinearities is proposed in Ref. [16], where nonlinear effects are seen as disturbances to the linear system and model parameters are calculated using the so-called disturbance rejection control theory. Applications of this approach to Coulomb friction identification in an industrial robot and crack detection in turborotors are reported in Refs. [16, 17]. A final example of grey-box modelling is the possibility of using an integral of the first kind to identify a nonlinear force, as investigated in the context of a computer disk drive servo in Ref. [18].

The present paper adopts a grey-box identification methodology by exploiting cubic splines to represent nonlinearities. A cubic spline is a continuous, piecewise third-order function defined by a series of knot points. The specific choice of splines of third order represents a good comprise between the flexibility of the fitted model, i.e. its ability to capture complex nonlinearities, and its parsimony, i.e. its quality to possess a low number of parameters. Spline-based identification of nonlinear systems has recently attracted some attention [19, 20, 21, 22]. Indeed, splines have the advantage of being as 
simple as ordinary polynomials, while overcoming some of their drawbacks. In particular, high-degree polynomials used to approximate complex nonlinearities are known to diverge rapidly outside the approximation interval, which generally prevents them from being utilised for numerical simulations. Furthermore, such high-degree polynomials may exhibit unbounded oscillations near the boundaries of the interval when interpolating equally-spaced knots. This behaviour, referred to as the Runge's phenomenon, indicates that increasing the degree of a polynomial interpolation does not always result in a decrease of the error. Finally, even if splines are naturally geared towards fitting smooth nonlinearities, they may also be reasonably well suited for representing piecewise linear functions [20], unlike polynomials. This latter capability requires non-evenly distributed knots, hence involving a nonlinear-in-the-parameters identification problem.

In this paper, cubic splines with equally-spaced knots are employed to identify the complex nonlinear dynamics of solar arrays in their stowed configuration. The estimation of the model parameters is achieved using the frequency-domain nonlinear subspace identification (FNSI) method, introduced in Ref. [23]. A distinct advantage of the FNSI approach is its capability to calculate accurately a large number of parameters, while maintaining an acceptable computational burden. This makes tractable the use of cubic splines to model nonlinearity in real-life mechanical systems, as the dimensionality of the inverse problem is known to increase dramatically in this case. The experimental structure of interest consists of two parallel aluminium plates assembled with bolted connections. This application is challenging because of the existence of impacts between the two plates at high excitation amplitude, and of the activation of complicated stiffness and damping mechanisms within the bolted connections.

The paper starts in Section 2 with an introduction to the theoretical background of the FNSI method. A detailed description of the solar array setup is provided in Section 3, and low-level data are exploited to derive a linear model. Three data sets collected for increasing force levels are then analysed in Section 4 in order to detect nonlinearity based on distortions appearing in frequency response function measurements. The nonlinear identification of the structure based on cubic splines is finally addressed in Section 5 using high-level data. The conclusions of the paper are summarised in Section 6 . 


\section{Nonlinear subspace identification in the frequency domain}

The FNSI method derives models of mechanical systems possessing localised nonlinearities directly from measured data, and without resorting to a preexisting numerical model, e.g. a finite element model [23]. It is applicable to multi-input, multi-output structures with high nonproportional damping and high modal density, and makes no assumption as to the importance of nonlinearity in the measured dynamics [24]. The vibrations of such nonlinear systems are governed by Newton's second law

$$
\mathbf{M} \ddot{\mathbf{q}}(t)+\mathbf{C} \dot{\mathbf{q}}(t)+\mathbf{K} \mathbf{q}(t)+\mathbf{g}(\mathbf{q}(t), \dot{\mathbf{q}}(t))=\mathbf{p}(t)
$$

where $\mathbf{M}, \mathbf{C}, \mathbf{K} \in \mathbb{R}^{n \times n}$ are the mass, linear viscous damping and linear stiffness matrices, respectively; $\mathbf{q}(t)$ and $\mathbf{p}(t) \in \mathbb{R}^{n}$ are the generalised displacement and external force vectors, respectively; $\mathbf{g}(t) \in \mathbb{R}^{n}$ is the essentially nonlinear, i.e. non-linearisable, restoring force vector encompassing elastic and dissipative contributions, and $n$ is the number of degrees of freedom (DOFs) of the structure obtained after spatial discretisation. The amplitude, direction, location and frequency content of the excitation $\mathbf{p}(t)$ determine in which regime, linear or nonlinear, the structure behaves. The effects of the $r$ lumped nonlinear components in the system are represented using a linear-in-the-parameters model of the form

$$
\mathbf{g}(\mathbf{q}(t), \dot{\mathbf{q}}(t))=\sum_{a=1}^{r} \sum_{b=1}^{s_{a}} c_{a, b} \mathbf{h}_{a, b}(\mathbf{q}(t), \dot{\mathbf{q}}(t)) .
$$

In this double sum, $s_{a}$ is the number of nonlinear basis functions $\mathbf{h}_{a, b}(\mathbf{q}(t), \dot{\mathbf{q}}(t))$ selected to describe the $a$-th nonlinearity, and $c_{a, b}$ are the associated coefficients. The total number of nonlinear basis functions introduced in the model is equal to $s=\sum_{a=1}^{r} s_{a}$. Linearity in the parameters avoids an iterative optimisation process, and issues related to initialisation and convergence thereof.

Given measurements of $\mathbf{p}(t)$ and $\mathbf{q}(t)$ or its derivatives, and an appropriate selection of the functionals $\mathbf{h}_{a, b}(t)$, the FNSI algorithm aims at computing estimates of (i) the frequency response function (FRF) matrix

$$
\mathbf{H}(\omega)=\left(-\omega^{2} \mathbf{M}+j \omega \mathbf{C}+\mathbf{K}\right)^{-1}
$$

describing the underlying linear properties of the system in Eq. (1), where $\omega$ is the frequency and $j$ the imaginary unit, and (ii) the nonlinear coefficients $c_{a, b}$ in Eq. (2).

The identification methodology essentially builds on a block-oriented interpretation of nonlinear structural dynamics, which sees nonlinearities as a feedback into the linear system in the forward loop [25], as illustrated in Fig. 1. This interpretation boils down to moving the nonlinear internal forces in Eq. (1) to the right-hand side, and viewing them as additional external forces applied to the underlying linear structure, that is

$$
\mathbf{M} \ddot{\mathbf{q}}(t)+\mathbf{C} \dot{\mathbf{q}}(t)+\mathbf{K} \mathbf{q}(t)=\mathbf{p}(t)-\sum_{a=1}^{r} \sum_{b=1}^{s_{a}} c_{a, b} \mathbf{h}_{a, b}(\mathbf{q}(t), \dot{\mathbf{q}}(t))
$$


[Figure 1 about here.]

Based on Eq. (4), the parameter estimation problem is solved in the frequency domain by processing a user-selected number of frequency lines in the measured band. A state-space representation of Eq. (4) is first extracted directly from frequency-domain data using robust tools from numerical analysis, including $\mathrm{QR}$ and singular value decompositions. The calculated state-space parameters are secondly converted into the modal space to estimate the underlying linear FRF matrix $\mathbf{H}(\omega)$, and to the physical space to estimate the nonlinear coefficients $c_{a, b}$. The knowledge of the output noise properties can be introduced in the algorithm to improve the accuracy of the estimates, as described Refs. [26, 27]. Fig. 2 presents an overview of the identification methodology. The interested reader is referred to Ref. [23] for a detailed introduction to the theoretical and practical aspects of the FNSI method. The demonstration of the FNSI method using numerical experiments was achieved in the case a real-life structure in Ref. [24]. The robustness of the method to various noise environments was also assessed therein using Monte-Carlo analyses.

[Figure 2 about here.] 


\section{Linear analysis of a simplified solar array structure at low excitation level}

As emphasised in the introductory section, nonlinear phenomena are frequently evidenced in dynamic testing of aerospace structures. In the particular case of space structures, nonlinearity commonly results from imperfect mounting interfaces between subsystems. For example, the Cassini spacecraft exhibited nonlinearities during a modal survey, arising from the existence of gaps in the truss supports of the Huygens probe [28]. The opening of a pin connection in the assembly of the International Space Station was similarly reported as a source of severe nonlinearity [29]. Nonlinear components may also be intentionally utilised in satellites, such as mechanical stops preventing the structural response from exceeding design specifications [30].

Another specific difficulty encountered in spacecraft testing is the behaviour of solar arrays in their stowed configuration. In fact, solar panels are folded in the launch vehicle fairing not only to save volume, but also to better withstand dynamic loads. When folded, they are assembled with bolts introduced at specific points, termed stacking points. Impact snubbers are also inserted between adjacent panels to maintain contact and absorb vibration. Some of the many phenomena that may lead to nonlinear effects are the loss of contact at high excitation amplitude, and complicated stiffness and damping mechanisms activated within the stacking points.

To investigate these phenomena, a simplified test rig that mimics the complex dynamics of folded solar generators was built at the FEMTO-ST Institute in Besançon, France. It consists of two $77 \times 44 \times 0.5 \mathrm{~cm}^{3}$ parallel aluminium plates mounted in free-free configuration, as pictured in Fig. 3 (a). They are clamped together at the top edge and connected through three stacking points (see Fig. $3(\mathrm{a}-\mathrm{b})$ ). Two solithane snubbers were mounted on steel supports at both corners of the bottom free edge, as shown in Fig. 3 (c). As pointed out in Ref. [31], the small contact areas between the stacking points and the plates are an additional possible source of nonlinear behaviour, as they may induce large, localised bending deformations.

In order to form the nonlinear basis functions $\mathbf{g}(\mathbf{q}(t), \dot{\mathbf{q}}(t))$ in Eq. (2), the FNSI method assumes the measurement of the relative displacement and relative velocity across all potentially nonlinear connections. The structure was therefore instrumented with ten accelerometers positioned on both sides of each suspected nonlinearity (see Fig. 3 (c)), i.e. the three stacking points and the two snubbers. These five connections are denoted NL $1-5$ in Fig. 3 (a). Excitation signals were applied to the front panel by means of a $10-N$ shaker, visible in Fig. 3 (a). Force and acceleration time histories were recorded at the excitation point through an impedance head. In this study, gaps of less than a millimetre were introduced between the two snubbers and the opposite plate such that there is no contact at rest (see Fig. $3(\mathrm{c}-\mathrm{d})$ ). However, the relatively small size of the sought gaps complicated the mounting of the two snubbers. This resulted in dissimilar gap sizes, and in an imperfect alignment of the snubber and the rear panel at NL 5, as noticeable in Fig. 3 (d). 
[Figure 3 about here.] 
Since a gap exists at rest between the two impact snubbers and the rear panel, a linear analysis of the structure can safely be achieved at very low level. To this end, a periodic noise forcing with a root-mean-squared (RMS) amplitude of $0.16 \mathrm{~N}$ was applied to the front panel, considering a sampling frequency of $6400 \mathrm{~Hz}$ and without feedback control of the amplitude level. A periodic noise consists in the repetition of a band-limited, random time series. A white amplitude spectrum in $5-350 \mathrm{~Hz}$ was chosen herein, as depicted in Fig. 4. The experiment was conducted over 28 periods of 4 seconds each, providing a frequency resolution of $0.25 \mathrm{~Hz}$. In a first pre-processing step, the first 10 periods were rejected since they were found to contain the transient response of the system. This ensures that no leakage distortions affect the transformation from time to frequency domain. In a second step, averaging was performed over the remaining 18 periods, which correspond to the steady-state response of the system, in order to mitigate noise and obtain a sample estimate of its covariance matrix [32].

[Figure 4 about here.]

The linear analysis of the structure is conducted using a typical stabilisation diagram [33, 34] charting the linear modal properties identified for increasing model orders, as proposed in Fig. 5 up to the order 150. This diagram was constructed using the FNSI algorithm considering no nonlinear basis function in input. The stabilisation thresholds in natural frequency, damping ratios and modal assurance criterion (MAC) were set to $2 \%, 10$ $\%$ and 0.95 , respectively. The knowledge of the output noise covariance matrix gained via the periodicity of the excitation can be incorporated in the stabilisation diagram, as illustrated in Fig. 6. This figure demonstrates that introducing a noise weighting into the subspace algorithm helps identify noisy modes, as particularly visible below $100 \mathrm{~Hz}$ where the signal-to-noise ratio (SNR) is low.

[Figure 5 about here.]

[Figure 6 about here.] 
The noise-weighted diagram in Fig. 6 leads to the selection of the order 108, for which 21 physical modes are captured in the $5-350 \mathrm{~Hz}$ band. Table 1 lists their natural frequencies and damping ratios. Damping ratios are all found to be lower than $1 \%$, as expected for an aluminium structure. Note that low-level data sets $(0.16 \mathrm{~N}$ RMS $)$ were regularly acquired during the entire test campaign to monitor the stability of the identified linear modal properties. This revealed the great stability of the assembly, in particular of the tightening torques in the stacking points. Fig. 7 (a $-\mathrm{f})$ depicts the deformed shapes of modes 1, 3, 7, 11, 12 and 15, respectively. Modes 1, 3 and 11 are likely to involve impacts between the two panels as they correspond to out-of-phase motions of their bottom free edges. Mode 2 possesses a deformed shape similar to mode 1 but, due to a lower dynamic amplification, it participates less in the solar panel response (see Fig. 9). One also points out an asymmetry around a vertical axis at mid-width of each panel for mode 1, entailing larger displacements at NL 1 and NL 4. A similar asymmetry is observed in Fig. 7 (c), where mode 7 is moreover seen to be an in-phase motion. Higher-frequency modes, such as modes 12 and 15 in Fig. 7 (e-f), are associated with in-plane deformations of the panels, causing large relative displacements at the stacking points, but no potential impact.

[Table 1 about here.]

[Figure 7 about here.]

The choice of the order 108 in the diagram of Fig. 6 is based on an individual analysis of its stabilisation columns. This is illustrated in Fig. $8(\mathrm{a}-\mathrm{d})$ where the frequency and damping ratio of modes 1 and 7 are depicted for increasing model orders. In general, the four graphs show important variations below the order 70, translating the successive appearance of stable modes in Fig. 6. Poles beyond the order 134 should also be assessed with care, because the accuracy of the subspace model decreases close to the maximum order. The motivation for selecting the order 108 is primarily due to the behaviour of the frequency and damping ratio of mode 1 in Fig. $8(\mathrm{a}-\mathrm{b})$. Indeed, they both exhibit a significant variability at lower orders, attributed to the reduced value of the SNR in the corresponding frequency interval. The complete stabilisation of mode 7 also requires the order 108 as the damping ratio in Fig. 8 (d) suddenly varies at orders 96 and 104, as a result of the late stabilisation of mode 2 in Fig. 6.

[Figure 8 about here.]

Fig. 9 finally shows the comparison between the FRF measured at NL 4 on the front panel (in black) and the corresponding FRF predicted by the linear subspace model (in dashed blue). In what follows, the difference between two frequency-dependent quantities should be understood as the difference of their magnitudes taken in $d B$. The difference between the noise variance and the measured FRF is plotted in Fig. 9 (in grey). This grey curve yields a convenient visualisation of the SNR with a negative sign. One notes that the SNR is around $40 d B$ inside the $100-350 \mathrm{~Hz}$ band, and reaches $60 \mathrm{~dB}$ at the resonance locations, which corresponds to the level of electrical and mechanical noise observed in 
typical experimental conditions. The low frequencies below $100 \mathrm{~Hz}$ are more importantly affected by noise as the SNR drops to $30 \mathrm{~dB}$. At very low frequencies, i.e. between 5 and $25 \mathrm{~Hz}$, the absence of dynamic amplification leads to a SNR of $10 \mathrm{~dB}$. Moreover, one observes a very good agreement between measured and synthesised FRFs. This is confirmed by the difference between the modelling error and the measured FRF depicted in Fig. 9 (in orange), showing a signal-to-modelling-error ratio of about $20 d B$ throughout the $100-350 \mathrm{~Hz}$ interval. Greater errors are noticed where the relative importance of noise is larger, in particular in the vicinity of anti-resonances and below $100 \mathrm{~Hz}$. It should be remarked that selecting the order 108 in Fig. 6 not only allows to estimate 21 physical poles in the input band, but also to include in the model some modes outside that band. This helps reconstruct accurately the linear FRFs of the system close to the cut-off frequency of the force, as clearly visible in Fig. 9.

[Figure 9 about here.]

\section{Nonlinearity detection at multiple excitation levels based on distortions in FRF measurements}

The comparison of FRFs computed using a linear estimator at different input levels is an interesting intermediate step in the nonlinear identification process. Since FRFs are required to be independent of the force amplitude spectrum if the system under test is linear [5], this comparison provides a reliable indicator of the presence of nonlinear behaviour in specific frequency bands. An additional interest in FRF measurements at multiple levels is that the complexity of the nonlinear modelling problem can be assessed prior to estimating parameters, by inspecting the global hardening or softening trend of the resonance peaks and the importance of their noisy-like distortions [32].

Fig. 10 depicts the FRFs measured at NL 4 on the front panel for input levels of 0.16 $N$ (in black), $1.91 N$ (in orange) and $3.79 N$ (in blue) RMS. The parameters of the excitation signals at 1.91 and $3.79 \mathrm{~N}$ RMS are identical to those selected at low level and discussed in Section 3. The analysis of Fig. 10 reveals substantial nonlinear effects throughout the excitation band. A close-up of the $5-100 \mathrm{~Hz}$ interval is proposed in Fig. 11. Modes 1 and 3 around 32 and $46 \mathrm{~Hz}$ are seen to be subjected to severe hardening distortions. This is confidently attributed to the activation of the impact snubbers, as Fig. $7(\mathrm{a}-\mathrm{b})$ showed that both modes involve out-of-phase motions of the two panels. The existence of impacts in the dynamics shifts the resonance frequencies of modes 1 and 3 of about 2 and $3 \mathrm{~Hz}$, respectively. Note that more accurate estimations of these frequency modifications will obtained in Section 5 (see Table 2). The more significant distortions affecting mode 3 are most probably due to a nonlinear geometrical effect originating from large, local deformations in the stacking points areas, as anticipated in Ref. [31]. The activation of such an effect in the structure of interest given a $10-N$ shaker is conditional upon two parameters, namely (i) the ratio between bolt and the panel sizes and (ii) the bolt tightening. Concerning the first parameter, the lower the ratio, the larger the local bending deformations, and hence the more important the resulting nonlinear distortions. 
In the present study, this ratio is of the order of magnitude of 0.1 (see Fig. $3(\mathrm{~b})$ ). Moreover, the three bolts at the stacking points of the assembly were tightened manually, leading to tightening torques of the order of magnitude of $1 \mathrm{~N} \mathrm{~m}$. The combination of these two parameters, i.e. a low bolt-to-panel ratio and a low tightening torque, with the substantial torsion deformations of the front panel associated with mode 3 in Fig. 7 (b) are found to involve geometrical nonlinearity for reasonable force levels of 1.91 and 3.79 $N$ RMS. One finally notes in Fig. 11 that mode 5 at $62 \mathrm{~Hz}$, which is an in-phase motion of the assembly, remains unaffected by an increase of the input level.

[Figure 10 about here.]

[Figure 11 about here.]

[Figure 12 about here.]

A second close-up in Fig. 12 displays the superposition of FRFs from 100 to $200 \mathrm{~Hz}$. Similarly to mode 5 , mode 7 at $145 \mathrm{~Hz}$ is invariant with respect to the force amplitude, in agreement with its deformed shape in Fig. 7 (c). Mode 10 at $165 \mathrm{~Hz}$, involving tensile deformations of the stacking connection at NL 2, manifests a decrease of the resonance frequency. This phenomenon, i.e. the softening due to micro-impacts of a bolted connection undergoing normal loadings, has often been reported in the technical literature $[35,36,37]$. In the present case study, it is made possible by the manual tightening of the bolts, similarly to the geometrical effects observed in Fig. 11. The deformed shape of mode 11 at $191 \mathrm{~Hz}$, presented in Fig. 7 (d), combines an out-ofphase motion of the panels and hence impacts, and tensile deformations at NL 2. This hardening-softening combination results in nonlinear distortions in Fig. 12, but without apparent modification of the resonance frequency.

A final close-up of the FRFs is plotted in $200-350 \mathrm{~Hz}$ in Fig. 13. In this interval, modal motions essentially consist in large relative displacements at the stacking points, as depicted in Fig. 7 (f) for mode 15. This translates into softening distortions of most resonance peaks in this band, associated with significant frequency shifts. For instance, the frequency of mode 19 approximately decreases from $339 \mathrm{~Hz}$ at low level to $335 \mathrm{~Hz}$ at $3.79 \mathrm{~N}$ RMS. This may be explained by the opening of the bolted connections at high frequency, implying an important loss of stiffness due to macro-impacts.

[Figure 13 about here.]

In summary, the analysis of the distortions affecting FRF measurements at multiple force levels in Figs. 10 to 13 highlighted very rich nonlinear dynamics. Impacts were first attested for modes entailing out-of-phase motions of the panels, e.g. modes 1 and 11, resulting in hardening behaviour. Large bending deformations localised in the stacking points areas were reported for mode 3 as an additional hardening phenomenon of geometrical nature. Softening effects due to tensile loadings of the bolted connections were finally evidenced, and attributed to micro-impacts in the case of mode 10 and gapping for high-frequency modes beyond $200 \mathrm{~Hz}$. 


\section{Nonlinearity identification at high excitation level using cubic splines}

According to Fig. 2, the construction of a nonlinear subspace model of the solar array dynamics at $3.79 N$ RMS requires the completion of five successive steps. The first step is an appropriate selection of the nonlinear basis functions $\mathbf{h}_{a, b}(\mathbf{q}(t), \dot{\mathbf{q}}(t))$ defined in Eq. (2). This is a difficult task as Section 4 revealed that the physical phenomena activating nonlinear behaviour in the structure are complex and numerous. In this context, the following section introduces an approximation strategy of nonlinear restoring forces relying on cubic splines, and bypassing the need for selecting the functional forms based on physics.

\subsection{Cubic spline representation of the solar array nonlinearities}

For the sake of simplicity and without loss of generality, the scope of this section is restricted to an elastic nonlinear restoring force $g(q(t))$ in the single-DOF case. Let $q$ be divided into $L$ segments of arbitrary length and defined by their abscissas, denoted by $q_{k}$ for $k=1, \ldots, L+1$. Each abscissa is associated with an ordinate $g_{k}$, together defining a knot $\left(q_{k}, g_{k}\right)$ of the spline. Thus, if $q$ is a displacement value in between knots $k$ and $k+1$, the corresponding point of a cubic-spline-based approximation of $g(q(t))$ is given by [38]

$g(q(t))=\left(2 t^{3}-3 t^{2}+1\right) g_{k}+\left(-2 t^{3}+3 t^{2}\right) g_{k+1}+\left(t^{3}-2 t^{2}+t\right)\left(q_{k+1}-q_{k}\right) g_{k}^{\prime}+\left(t^{3}-t^{2}\right)\left(q_{k+1}-q_{k}\right) g_{k+1}^{\prime}$

where $t$ is the normalised displacement $\frac{q-q_{k}}{q_{k+1}-q_{k}}$. The computation of the first derivatives $g_{k}^{\prime}=\partial g_{k} / \partial q_{k}$ can be achieved by forcing the cubic spline and its first two derivatives to be continuous across each of the interior knots. This results in $L-1$ linear constraint equations

$\frac{g_{k-1}^{\prime}}{q_{k}-q_{k-1}}+2\left(\frac{1}{q_{k}-q_{k-1}}+\frac{1}{q_{k+1}-q_{k}}\right) g_{k}^{\prime}+\frac{g_{k+1}^{\prime}}{q_{k+1}-q_{k}}=3\left(\frac{g_{k}-g_{k-1}}{\left(q_{k}-q_{k-1}\right)^{2}}+\frac{g_{k+1}-g_{k}}{\left(q_{k+1}-q_{k}\right)^{2}}\right)$.

Since the essentially nonlinear restoring force $g(q(t))$ is zero and has zero slope at equilibrium, one should also enforce, in the segment containing the abscissa of the equilibrium point, that

$$
\left(t_{0}^{3}-2 t_{0}^{2}+t_{0}\right)\left(q_{k+1}-q_{k}\right) g_{k}^{\prime}+\left(t_{0}^{3}-t_{0}^{2}\right)\left(q_{k+1}-q_{k}\right) g_{k+1}^{\prime}=-\left(2 t_{0}^{3}-3 t_{0}^{2}+1\right) g_{k}-\left(-2 t_{0}^{3}+3 t_{0}^{2}\right) g_{k+1}
$$

and

$$
\left(3 t_{0}^{2}-4 t_{0}+1\right)\left(q_{k+1}-q_{k}\right) g_{k}^{\prime}+\left(3 t_{0}^{2}-2 t_{0}\right)\left(q_{k+1}-q_{k}\right) g_{k+1}^{\prime}=6\left(t_{0}-t_{0}^{2}\right)\left(g_{k}-g_{k+1}\right)
$$

where $t_{0}=\frac{-q_{k}}{q_{k+1}-q_{k}}$. Eqs (6), (7) and (8) constitute $L+1$ relations that uniquely define the $L+1$ parameters $g_{1}^{\prime}, \ldots, g_{L+1}^{\prime}$ as functions of the ordinates of the knots $g_{1}, \ldots, g_{L+1}$. The first derivatives can thus be substituted in Eq. (5) to compute the basis functions associated with the ordinates. These basis functions correspond to the terms $\mathbf{h}_{a, b}(t)$ introduced as additional external forces in the FNSI algorithm, as described in Section 2. 
In the solar array identification, splines in displacement and velocity are implemented at NL 4 and NL 5. No nonlinearity is considered at NL 1, NL 2 and NL 3 since the relative displacements and velocities measured at these three connections are of comparable amplitude to the noise level. This is illustrated in Fig. $14(\mathrm{a}-\mathrm{b})$ where the relative displacements at NL 1 and NL 4 are plotted versus time and compared to the corresponding noise signal. The SNR in RMS value is equal to 11.65 at NL 4, while it drops to 1.51 at NL 1 because of the position of the shaker and of the stiffness distribution of the assembly. Note that implementing splines exclusively at NL 4 and NL 5 implies that the five nonlinear connections in the structure, namely the three stacking points and the two impact snubbers, are modelled using only two equivalent nonlinear springs and two equivalent nonlinear dampers. This prevents the effects of these connections from being distinguished in the resulting elastic and dissipative force curves (see the analysis of Figs. 20 and 21 in Section 5.5).

[Figure 14 about here.]

The optimal number of knots in the definition of the splines should rigorously be sought by minimising the difference in some metric between the predictions of the nonlinear model and measured data. In practice, an acceptable number can be found by trial and error, maximising the accuracy of the underlying linear properties of the system estimated from nonlinear data. Splines with 5 and 3 knots are therefore chosen to model stiffness and damping nonlinearities, respectively, because the agreement between the properties listed in Table 1 and the corresponding estimates computed at $3.79 \mathrm{~N}$ RMS was found to degrade for larger numbers of knots. It is worth stressing the high dimensionality of the consequent inverse problem, which encompasses 17 inputs, i.e. 1 external force and 16 nonlinear basis functions, and 11 outputs. Owing to this dimensionality, time-domain identification techniques, in particular the subspace method proposed in Ref. [39], are likely to suffer from computational memory issues, because of their inability to reject less informative measured samples (see Section 5.2). Moreover, few methods in the technical literature are capable of reliably handling a large amount of nonlinear coefficients. For instance, the conditioned reverse path method [40] estimates the coefficients sequentially, i.e. errors are accumulated throughout the identification process, and would probably lead to very large errors in the present case study. Another example is the nonlinear identification through feedback of the outputs method [25], whose estimation accuracy is known to decrease rapidly as the number of parameters increases.

\subsection{Choice of the processed bandwidth}

The FRF calculated at NL 4 on the front panel over $0-1500 \mathrm{~Hz}$ is depicted in Fig. 15 (in black), together with the difference between the noise variance and the FRF (in grey). The SNR inside the input band reaches 70 to $80 d B$ at the resonance locations, compared to $60 \mathrm{~dB}$ at $0.16 \mathrm{~N}$ RMS (see Fig. 9). The frequencies below $100 \mathrm{~Hz}$ still suffer from larger noise distortions with a SNR around $50 \mathrm{~dB}$. The bandwidth processed in the identification is restricted to $1500 \mathrm{~Hz}$ where the SNR is close to $0 \mathrm{~dB}$. This choice completes the 
second step of the FNSI methodology. The restriction of the bandwidth helps avoid computational memory issues that may arise due to the aforementioned dimensionality of the inverse problem, while preserving the nonlinear distortions appearing outside the input band in the estimation of model parameters.

[Figure 15 about here.]

\subsection{Determination of the model order}

The next step in Fig. 2 is the determination of the model order. In the presence of nonlinearities, the model order translates the number of underlying linear modes excited in the output data [23]. This implies that, similarly to linear system identification, a stabilisation analysis can be utilised as decision-making tool [24]. The stabilisation diagram plotted in Fig. 16 indicates that 20 physical modes of the structure can be incorporated in a model of order 130. The choice of the order 130 is motivated by the study of the natural frequency and damping ratio of modes 1 and 3, represented in Fig. $17(\mathrm{a}-\mathrm{d})$ for increasing model orders. One observes that their estimates calculated at low level in Section 3 cannot be accurately recovered with acceptable stabilisation features at lower orders. Note that mode 2 identified at low level is not recovered at $3.79 \mathrm{~N}$ RMS, most probably because it hardly participates in the response and is dominated by the nonlinear distortions affecting modes 1 and 3 (see Fig. 11).

[Figure 16 about here.]

[Figure 17 about here.]

\subsection{Estimation of the underlying linear properties}

[Table 2 about here.]

Table 2 lists the linear frequencies of the structure identified at low level (see Section 3) for a restricted number of representative modes (second column), together with the associated frequencies estimated at high level using splines (third column). A good agreement is noted for modes below $200 \mathrm{~Hz}$. In particular, the frequencies of modes 1 and 3 are correctly predicted, while they were shown to suffer from the most significant nonlinear distortions in Section 4. This is confirmed in Fig. 18 where a comparison between the FRF measured at low level at NL 4 on the front panel and the FRF synthesised using FNSI at high level is plotted. Table 2 and Fig. 18 prove that the impacts at the snubber connections and the large bending deformations at the stacking points are correctly represented in the nonlinear model.

[Figure 18 about here.] 
By contrast, the frequencies of the modes between 200 and $350 \mathrm{~Hz}$ are not correctly identified, meaning that the softening effects affecting high-frequency modes are not captured. This is illustrated in Fig. 19, where the linear FRF calculated at NL 4 on the front panel (in black) is compared to the FRF synthesised at high level by the FNSI algorithm (in dashed blue) over $200-350 \mathrm{~Hz}$. The FRF computed at high level using a linear estimator is also plotted (in orange). One observes that the subspace model linearises the high-frequency dynamics by fitting the distorted resonance peaks in opposition with the first objective of the FNSI method listed in Section 2, namely the synthesis of the underlying linear FRFs of the system. This is also evidenced in the fourth column of Table 2 where the frequencies estimated using a linear model fitted to nonlinear data are given.

[Figure 19 about here.]

[Table 3 about here.]

The fifth column of Table 2 finally lists the frequencies estimated using ordinary polynomials possessing the same number of parameters as the cubic splines defined in Section 5.1. Specifically, essentially nonlinear polynomials of order 6 were chosen for stiffness nonlinearities, and fourth-order polynomials for damping nonlinearities. In the $200-350 \mathrm{~Hz}$ band, the polynomial model brings no improvement with respect to the spline model, i.e. it also yields linearised frequencies. This shows that the underestimation of the frequencies beyond $200 \mathrm{~Hz}$ is not to be attributed to inaccuracies in the estimation of parameters, but to the inability of continuous functions like splines and polynomials to capture micro-impacts and gapping, most likely associated with discontinuous restoring forces. Moreover, modes 1 and 3 are less accurately retrieved by the polynomials, proving that splines offer additional flexibility in the representation of complex nonlinearities. This is also clearly demonstrated in Table 3 where the estimation of the linear damping ratios is assessed using the spline and polynomial models. In particular, the estimates obtained for modes 1 and 3 using polynomials are greatly improved by exploiting splines.

\subsection{Estimation of the nonlinear restoring force curves}

The last step in Fig. 2 is the conversion of the state-space model to the physical space in order to visualise the restoring forces fitted using cubic splines and polynomials. To this end, the conversion scheme detailed in Ref. [23] is utilised, limiting the frequency interval of conversion to $200 \mathrm{~Hz}$ since the two models do not perform well for higher frequencies. The elastic force curves at NL 4 and NL 5 are proposed in Fig. $20(\mathrm{a}-\mathrm{b})$. One first notes that the spline- and polynomial-based curves give rise to similar dynamics. They exhibit a significant, yet smooth, increase of stiffness for negative displacements, translating impacts on the snubbers. Two variations of slope in the impact region are seen in Fig. 20 (b) resulting from the misalignment between the snubber at NL 5 and the rear panel, pointed out in Fig. 3 (d). Moreover, the curves present an increase of stiffness in positive displacement caused by the geometrically nonlinear deformations in 
the stacking points areas. The associated nonlinear coefficient is found to be larger at NL 5. A similar observation, but in terms of linear stiffness coefficients, was made in the analysis of the deformed shapes of modes 1 and 7 in Fig. 7 (a, c). It should be noted that, though being similar qualitatively, the two curves in Fig. 20 (a-b) are not identical, translating an asymmetry between NL 4 and NL 5. This asymmetry is due to (i) the manual tightening of the stacking points which is likely to be different from one connection to another, and (ii) to the aforementioned misalignment of the snubber and the real panel at NL 5 .

Finally, the dissipative force curves at NL 4 and NL 5 are depicted in Fig. $21(\mathrm{a}-\mathrm{b})$. Nonlinear damping mainly affects NL 4 through positive even and odd terms. The corresponding spline curve at NL 5 shows an even contribution with an amplitude three times lower than the force curve in Fig. 21 (a). It should be stressed that the identification of nonlinear damping forces remains a challenging task because of their marginal amplitude with respect to stiffness forces. Moreover, the complete representation of the nonlinear damping effects in the solar panel structure might require a more complicated model than the nonlinear viscous damping functional $\mathbf{g}(\dot{\mathbf{q}}(t))$.

[Figure 20 about here.]

[Figure 21 about here.]

\section{Conclusion}

The objective of the present paper was to investigate the usefulness of cubic splines to represent complex stiffness and damping nonlinearities based on real-life data. For that purpose, splines were embedded in a nonlinear subspace identification algorithm capable of handling high-dimensional inverse problems, referred to as the FNSI method. Splines were shown to bring superior accuracy with respect to ordinary polynomials in the identification of the linear and nonlinear properties of a space solar array structure in stowed configuration. In particular, nonlinear effects due to unilateral impacts and geometrically nonlinear deformations were successfully captured in the nonlinear model. This work proves that the joint utilisation of splines and FNSI paves the way for addressing large-scale structures exhibiting non-intuitive nonlinearities.

\section{Acknowledgements}

The author J.P. Noël is a Research Fellow (FRIA fellowship) of the Fonds de la Recherche Scientifique - FNRS which is gratefully acknowledged. 


\section{References}

[1] J.R. Ahlquist, J.M. Carreño, H. Climent, R. de Diego, and J. de Alba. Assessment of nonlinear structural response in A400M GVT. In Proceedings of the 28th International Modal Analysis Conference (IMAC), Jacksonville, FL, 2010.

[2] M. Link, M. Boeswald, S. Laborde, M. Weiland, and A. Calvi. Non-linear experimental modal analysis and application to satellite vibration test data. In Proceedings of the 3rd Conference on Computational Methods in Structural Dynamics and Earthquake Engineering (COMPDYN), Corfu, Greece, 2011.

[3] A. Carrella and D.J. Ewins. Identifying and quantifying structural nonlinearities in engineering applications from measured frequency response functions. Mechanical Systems and Signal Processing, 25:1011-1027, 2011.

[4] J.P. Noël, L. Renson, G. Kerschen, B. Peeters, S. Manzato, and J. Debille. Nonlinear dynamic analysis of an F-16 aircraft using GVT data. In Proceedings of the International Forum on Aeroelasticity and Structural Dynamics (IFASD), Bristol, UK, 2013.

[5] G. Kerschen, K. Worden, A.F. Vakakis, and J.C. Golinval. Past, present and future of nonlinear system identification in structural dynamics. Mechanical Systems and Signal Processing, 20:505-592, 2006.

[6] J. Sjöberg, Q. Zhang, L. Ljung, A. Benveniste, B. Delyon, P.Y. Glorennec, H. Hjalmarsson, and A. Juditsky. Nonlinear black-box modeling in system identification: a unified overview. Automatica, 31(12):1691-1724, 1995.

[7] G. Kerschen, V. Lenaerts, and J.C. Golinval. Identification of a continuous structure with a geometrical non-linearity. Part I: Conditioned reverse path method. Journal of Sound and Vibration, 262:889-906, 2003.

[8] S.A. Billings. Nonlinear System Identification: NARMAX Methods in the Time, Frequency, and Spatio-Temporal Domains. Wiley, New York, 2013.

[9] K. Worden, W.J. Staszewski, and J.J. Hensman. Natural computing for mechanical systems research: A tutorial overview. Mechanical Systems and Signal Processing, $25: 4-111,2011$.

[10] J. Paduart, L. Lauwers, J. Swevers, K. Smolders, J. Schoukens, and R. Pintelon. Identification of nonlinear systems using polynomial nonlinear state space models. Automatica, 46:647-656, 2010.

[11] K. Worden, C.X. Wong, U. Parlitz, A. Hornstein, D. Engster, T. Tjahjowidodo, F. Al-Bender, D.D. Rizos, and S.D. Fassois. Identification of pre-sliding and sliding friction dynamics: Grey box and black-box models. Mechanical Systems and Signal Processing, 21:514-534, 2007. 
[12] M.F. Platten, J.R. Wright, J.E. Cooper, and G. Dimitriadis. Identification of a nonlinear wing structure using an extended modal model. Journal of Aircraft, 46(5):1614-1626, 2009.

[13] S.F. Masri and T.K. Caughey. A nonparametric identification technique for nonlinear dynamic problems. Journal of Applied Mechanics, 46:433-447, 1979.

[14] S.F. Masri, A.G. Chassiakos, and T.K. Caughey. Structure-unknown non-linear dynamic systems: identification through neural networks. Smart Materials and Structures, 1:45-56, 1992.

[15] Y. Fan and C.J. Li. Non-linear system identification using lumped parameter models with embedded feedforward neural networks. Mechanical Systems and Signal Processing, 16(2-3):357-372, 2002.

[16] P.C. Müller. Indirect measurements of nonlinear effects by state observers. In Proceedings of the IUTAM Symposium on Nonlinear Dynamics in Engineering Systems, Stuttgart, Germany, 1989.

[17] D. Söffker, J. Bajkowski, and P.C. Müller. Detection of cracks in turborotors - a new observer based method. ASME Journal of Dynamic Systems, Measurement, and Control, 115:518-524, 1993.

[18] W. Messner and R. Horowitz. Identification of a nonlinear function in a dynamical system. ASME Journal of Dynamic Systems, Measurement, and Control, 115:587591, 1993.

[19] S. Guarnieri, F. Piazza, and A. Uncini. Multilayer feedforward networks with adaptive spline activation function. IEEE Transactions on Neural Networks, 10:672-683, 1999.

[20] E.J. Dempsey and D.T. Westwick. Identification of Hammerstein models with cubic spline nonlinearities. IEEE Transactions on Biomedical Engineering, 51(2):237-245, 2004.

[21] K. Jalaleddini, D.T. Westwick, and R.E. Kearney. Subspace identification of Hammerstein systems using B-splines. In Proceedings of the 34th Annual International Conference of the IEEE EMBS, San Diego, CA, 2012.

[22] Y.C. Zhu. Parametric Wiener model identification for control. In Proceedings of IFAC World Congress, Beijing, China, 1999.

[23] J.P. Noël and G. Kerschen. Frequency-domain subspace identification for nonlinear mechanical systems. Mechanical Systems and Signal Processing, 40:701-717, 2013.

[24] J.P. Noël, S. Marchesiello, and G. Kerschen. Subspace-based identification of a nonlinear spacecraft in the time and frequency domains. Mechanical Systems and Signal Processing, 43:217-236, 2014. 
[25] D.E. Adams and R.J. Allemang. A frequency domain method for estimating the parameters of a non-linear structural dynamic model through feedback. Mechanical Systems and Signal Processing, 14:637-656, 2000.

[26] T. McKelvey, H. Akçay, and L. Ljung. Subspace-based multivariable system identification from frequency response data. IEEE Transactions on Automatic Control, 41(7):960-979, 1996.

[27] R. Pintelon. Frequency-domain subspace system identification using non-parametric noise models. Automatica, 38:1295-1311, 2002.

[28] K. Carney, I. Yunis, K. Smith, and C.Y. Peng. Nonlinear dynamic behavior in the Cassini spacecraft modal survey. In Proceedings of the 15th International Modal Analysis Conference (IMAC), Orlando, FL, 1997.

[29] M. Laible, K. Fitzpatrick, and M. Grygier. International Space Station 2A array modal analysis. In Proceedings of the 31st International Modal Analysis Conference (IMAC), Garden Grove, CA, 2013.

[30] J.P. Noël, L. Renson, and G. Kerschen. Experimental identification of the complex dynamics of a strongly nonlinear spacecraft structure. In Proceedings of the ASME International Design Engineering Technical Conferences (IDETC), Portland, OR, 2013.

[31] A. Hot, G. Kerschen, E. Foltête, and S. Cogan. Detection and quantification of nonlinear structural behaviour using principal component analysis. Mechanical Systems and Signal Processing, 26:104-116, 2012.

[32] R. Pintelon and J. Schoukens. System Identification: A Frequency Domain Approach. Wiley-Blackwell, 2012.

[33] B. Peeters and G. de Roeck. Reference-based stochastic subspace identification for output-only modal analysis. Mechanical Systems and Signal Processing, 13(6):855878, 1999.

[34] P.G. Bakir. Automation of the stabilization diagrams for subspace based system identification. Expert Systems with Applications, 38:14390-14397, 2011.

[35] C.J. Hartwigsen, Y. Song, D.M. McFarland, L.A. Bergman, and A.F. Vakakis. Experimental study of non-linear effects in a typical shear lap joint configuration. Journal of Sound and Vibration, 277:327-351, 2004.

[36] H. Ahmadian and H. Jalali. Identification of bolted lap joints parameters in assembled structures. Mechanical Systems and Signal Processing, 21:1041-1050, 2007.

[37] M. Eriten, M. Kurt, G. Luo, D.M. McFarland, L.A. Bergman, and A.F. Vakakis. Nonlinear system identification of frictional effects in a beam with a bolted joint connection. Mechanical Systems and Signal Processing, 39:245-264, 2013.

[38] C. De Boor. A Practical Guide to Splines. Springer-Verlag, New York, 1978. 
[39] S. Marchesiello and L. Garibaldi. A time domain approach for identifying nonlinear vibrating structures by subspace methods. Mechanical Systems and Signal Processing, 22:81-101, 2008.

[40] C.M. Richards and R. Singh. Identification of multi-degree-of-freedom non-linear systems under random excitations by the reverse-path spectral method. Journal of Sound and Vibration, 213:673-708, 1998. 


\section{List of Figures}

1 Feedback interpretation of nonlinear structural dynamics [25]. . . . . . . . 23

2 Overview of the FNSI methodology. . . . . . . . . . . . . . . 24

3 Simplified experimental solar array structure in stowed configuration. . . . 25

4 Amplitude spectrum over $0-1500 \mathrm{~Hz}$ of a single period of the noise forcing at $0.16 N$ RMS. . . . . . . . . . . . . . . . . . . . 26

5 Stabilisation diagram computed at low level (0.16 N RMS) without noise weighting. Cross: stabilisation in natural frequency; square: extra stabilisation in damping ratio; circle: extra stabilisation in MAC; triangle: full stabilisation. . . . . . . . . . . . . . . . 27

6 Stabilisation diagram computed at low level (0.16 N RMS) with noise weighting. The selected order is indicated using a blue line. Crosses, squares, circles and triangles are defined similarly to Fig. 5. . . . . . . . .

7 Deformed shapes corresponding to modes 1, 3, 7, 11, 12 and 15 identified at low level (0.16 $N$ RMS). Geometrical dimensions are given in centimetres. 29

8 Frequency and damping ratio of $(\mathrm{a}-\mathrm{b})$ mode 1 and $(\mathrm{c}-\mathrm{d})$ mode 7 calculated at low level (0.16 $N$ RMS ) for increasing model orders. The selected order is indicated using blue lines. . . . . . . . . . . . . . . . .

$9 \quad$ FRF measured at low level $(0.16 N$ RMS) at NL 4 on the front panel (in black) and corresponding FRF predicted by the linear subspace model (in dashed blue) over $5-350 \mathrm{~Hz}$; difference between the noise variance and the measured FRF (in grey); difference between the modelling error and the measured FRF (in orange). . . . . . . . . . . . . . . . 31

10 FRFs measured at NL 4 on the front panel over $5-350 \mathrm{~Hz}$ at $0.16 \mathrm{~N}$ (in black), $1.91 N$ (in orange) and $3.79 N$ (in blue) RMS. . . . . . . . . . .

11 Close-up in the $5-100 \mathrm{~Hz}$ band of the FRFs measured at NL 4 on the front panel at $0.16 N$ (in black), $1.91 N$ (in orange) and $3.79 N$ (in blue) RMS. . . . . . . . . . . . . . . . . . . .

12 Close-up in the $100-200 \mathrm{~Hz}$ band of the FRFs measured at NL 4 on the front panel at $0.16 N$ (in black), $1.91 N$ (in orange) and $3.79 N$ (in blue)

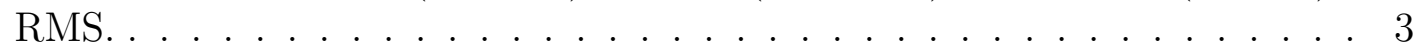

13 Close-up in the $200-350 \mathrm{~Hz}$ band of the FRFs measured at NL 4 on the front panel at $0.16 N$ (in black), $1.91 N$ (in orange) and $3.79 N$ (in blue) RMS. . . . . . . . . . . . . . . . . . .

14 Relative displacement at (a) NL 1 and (b) NL 4 plotted versus time and compared to the associated noise signal. 
15 FRF calculated at high level (3.79 $N$ RMS $)$ at NL 4 on the front panel over $0-1500 \mathrm{~Hz}$ (in black), and difference between the noise variance and the FRF (in grey). . . . . . . . . . . . . . . . . . . . 37

16 Stabilisation diagram computed at high level (3.79 $N$ RMS) with noise weighting. The selected order is indicated using a blue line. Crosses, squares, circles and triangles are defined similarly to Fig. 5 . . . . . . . . . 38

17 Linear frequency and damping ratio of modes $1(\mathrm{a}-\mathrm{b})$ and $3(\mathrm{c}-\mathrm{d})$ calculated at high level (3.79 $\mathrm{N}$ RMS) for increasing model orders. The selected order is indicated using blue lines. . . . . . . . . . . . . . . . . 39

18 Comparison between the FRF measured at low level (0.16 $N$ RMS $)$ at NL 4 on the front panel over $5-100 \mathrm{~Hz}$ (in black), and the FRF synthesised at high level (3.79 $\mathrm{N}$ RMS) by the FNSI algorithm (in dashed blue). . . . 40

19 Comparison between the FRF measured at low level (0.16 N RMS) at NL 4 on the front panel over $200-350 \mathrm{~Hz}$ (in black), and the FRF reconstructed at high level (3.79 $N$ RMS) by the FNSI algorithm (in dashed blue); the FRF measured at high level (3.79 $N$ RMS) is also plotted (in orange). . . . 41

20 Nonlinear stiffness curve constructed at high level (3.79 $N$ RMS) using cubic splines (black circles) and sixth-order polynomials (blue dots): (a) NL 4; (b) NL 5. Orange squares localise the 5 chosen knots of the splines. .

21 Nonlinear damping curve constructed at high level (3.79 $N$ RMS) using cubic splines (black circles) and fourth-order polynomials (blue dots): (a) NL 4; (b) NL 5. Orange squares localise the 3 chosen knots of the splines. . 


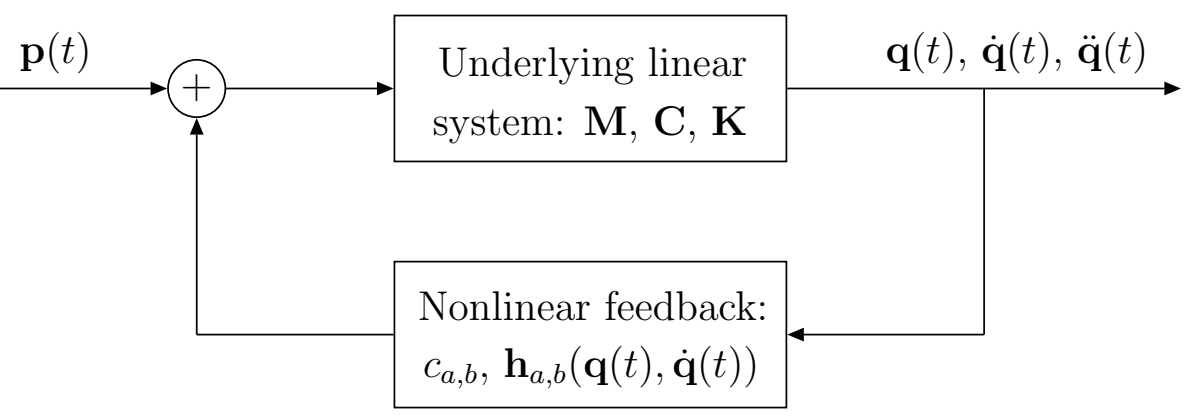

Figure 1: Feedback interpretation of nonlinear structural dynamics [25]. 
1. Select appropriate basis functions $\mathbf{h}_{a, b}(\mathbf{q}(t), \dot{\mathbf{q}}(t))$ to represent the nonlinearities.

2. Choose the number of processed frequency lines in the measured band.

3. Determine the order of the state-space representation of Eq. (4) and calculate its parameters from frequency-domain data.

4. Convert the state-space model to modal space to estimate the underlying linear FRF matrix $\mathbf{H}(\omega)$.

5. Convert the state-space model to physical space to estimate the nonlinear coefficients $c_{a, b}$.

Figure 2: Overview of the FNSI methodology. 


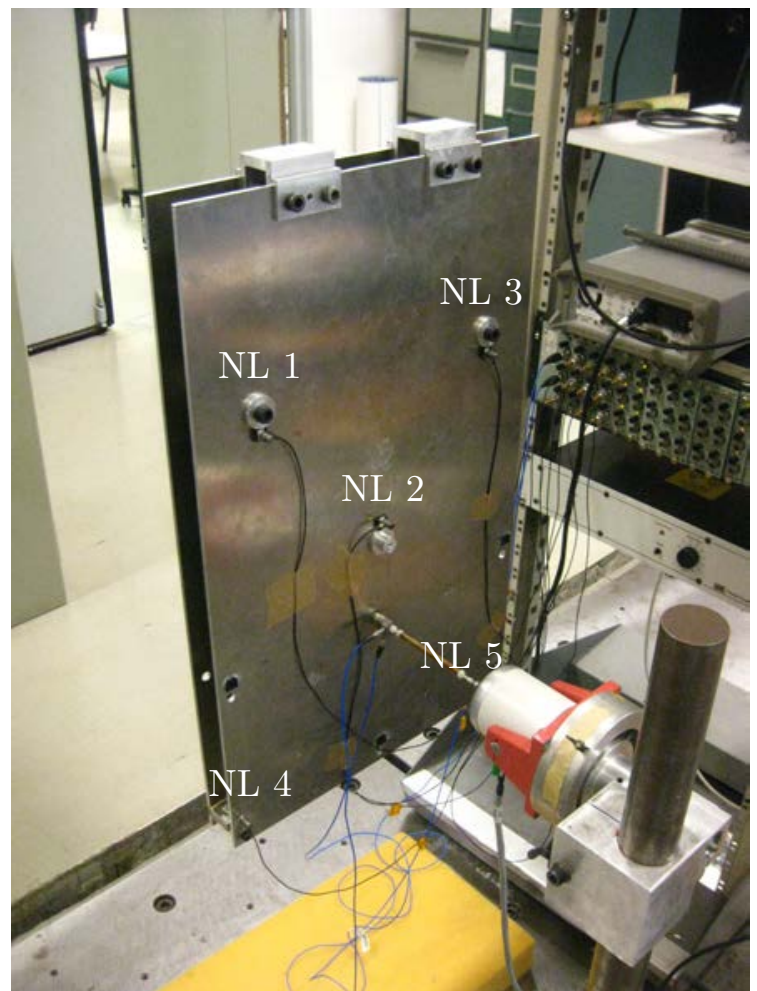

(a) Overall view of the two aluminium plates clamped together at the top edge, and mounted in free-free configuration.

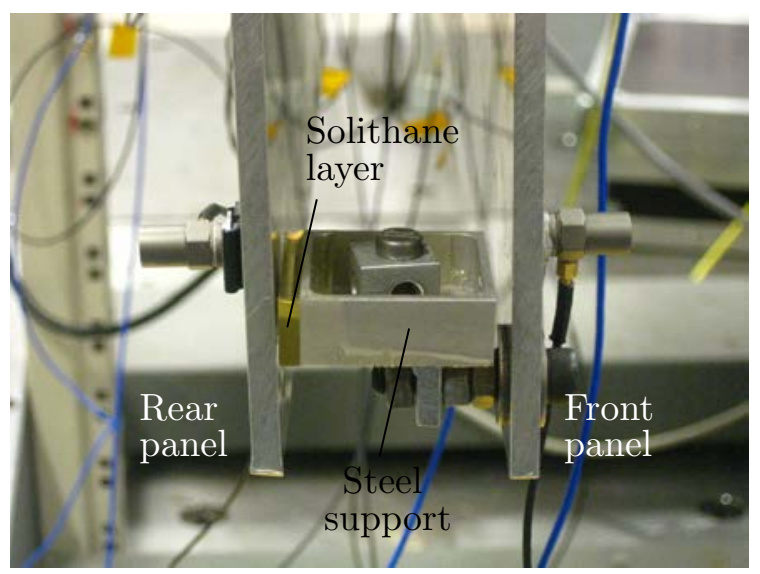

(c) Close-up of the impact snubber at NL 4 instrumented with one accelerometer on both sides.

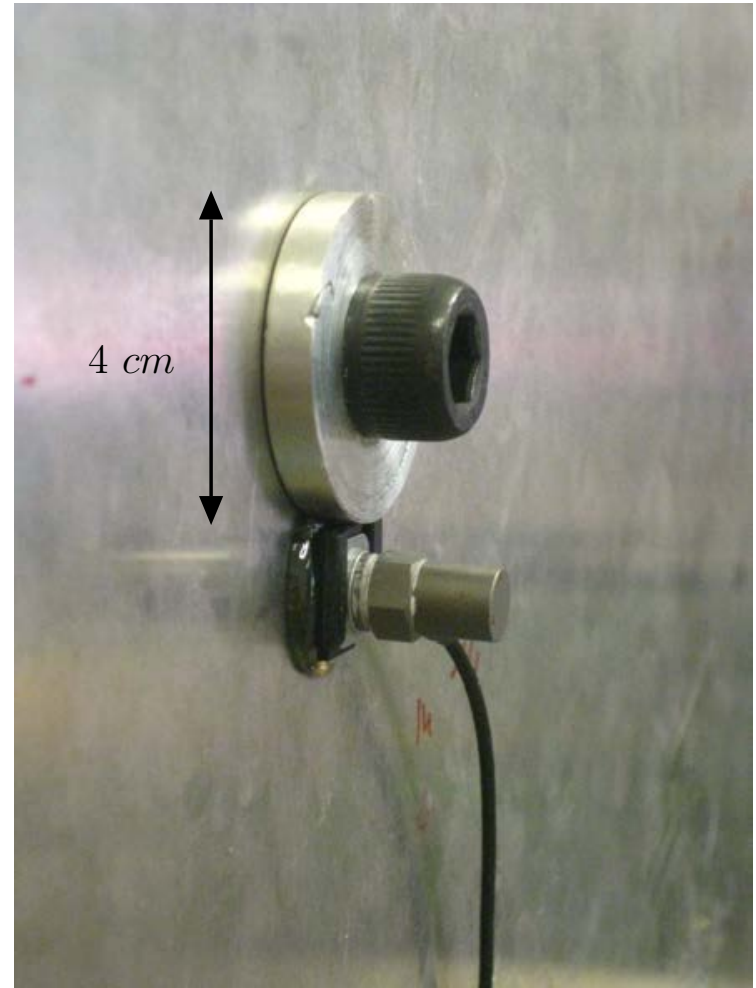

(b) Close-up of the stacking point at NL 1.

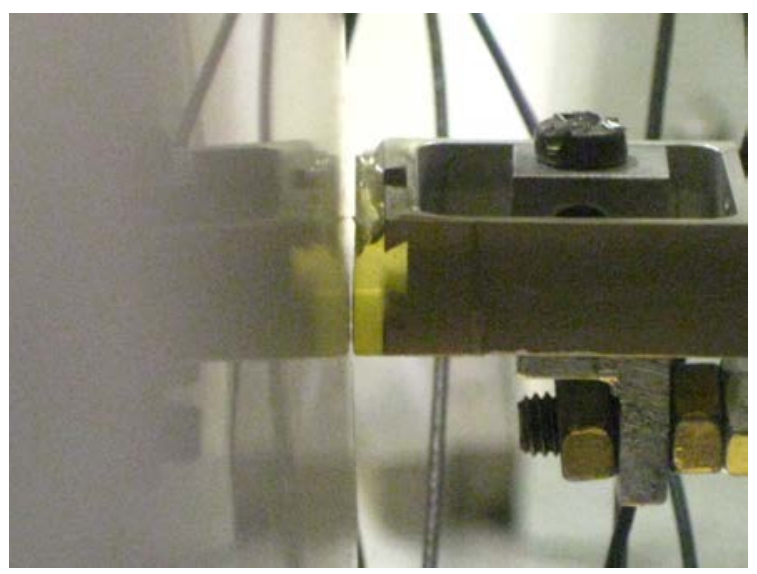

(d) Close-up at NL 5 showing the imperfect alignment between the snubber and the rear panel.

Figure 3: Simplified experimental solar array structure in stowed configuration. 


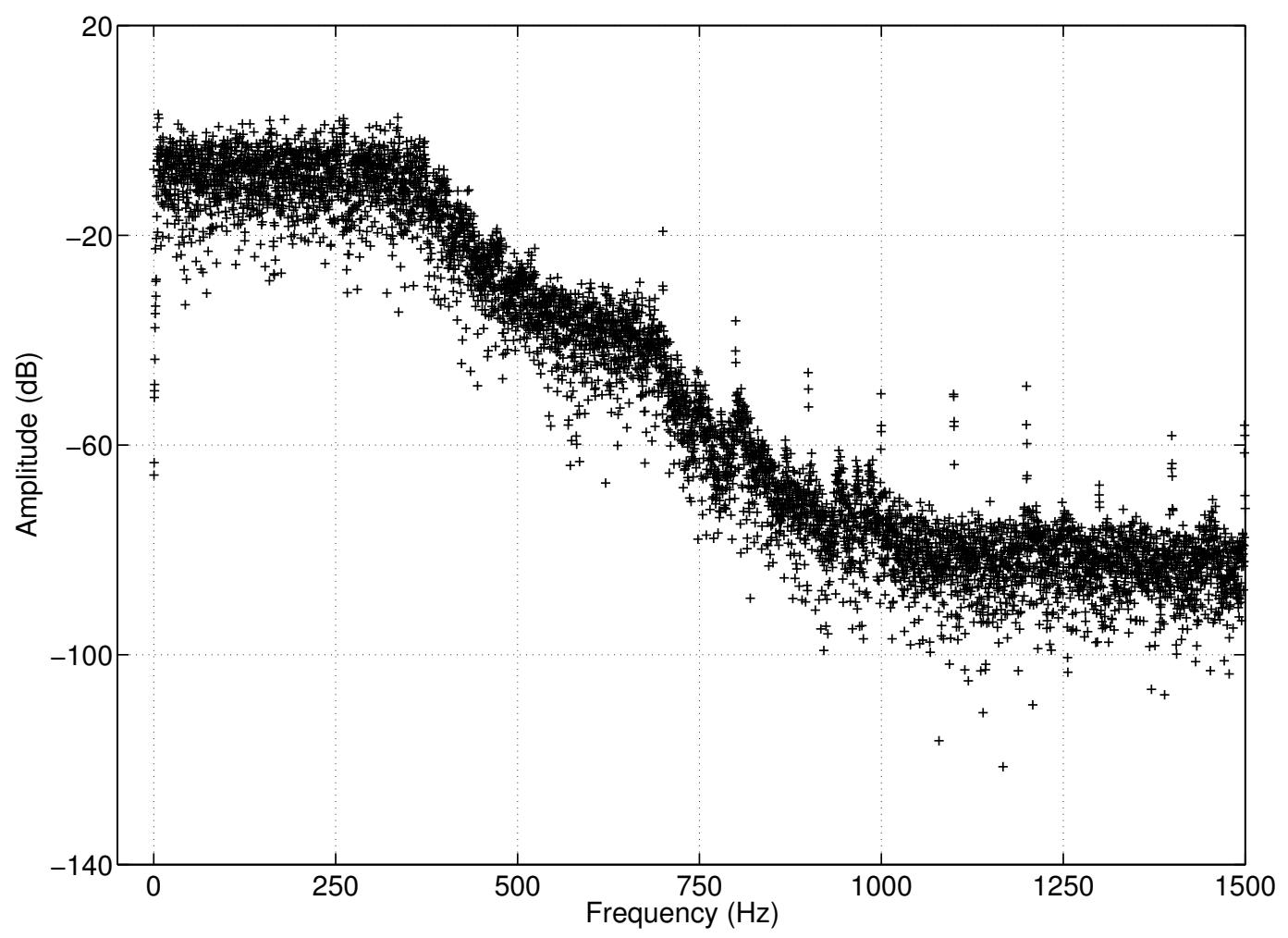

Figure 4: Amplitude spectrum over $0-1500 \mathrm{~Hz}$ of a single period of the noise forcing at $0.16 N$ RMS. 


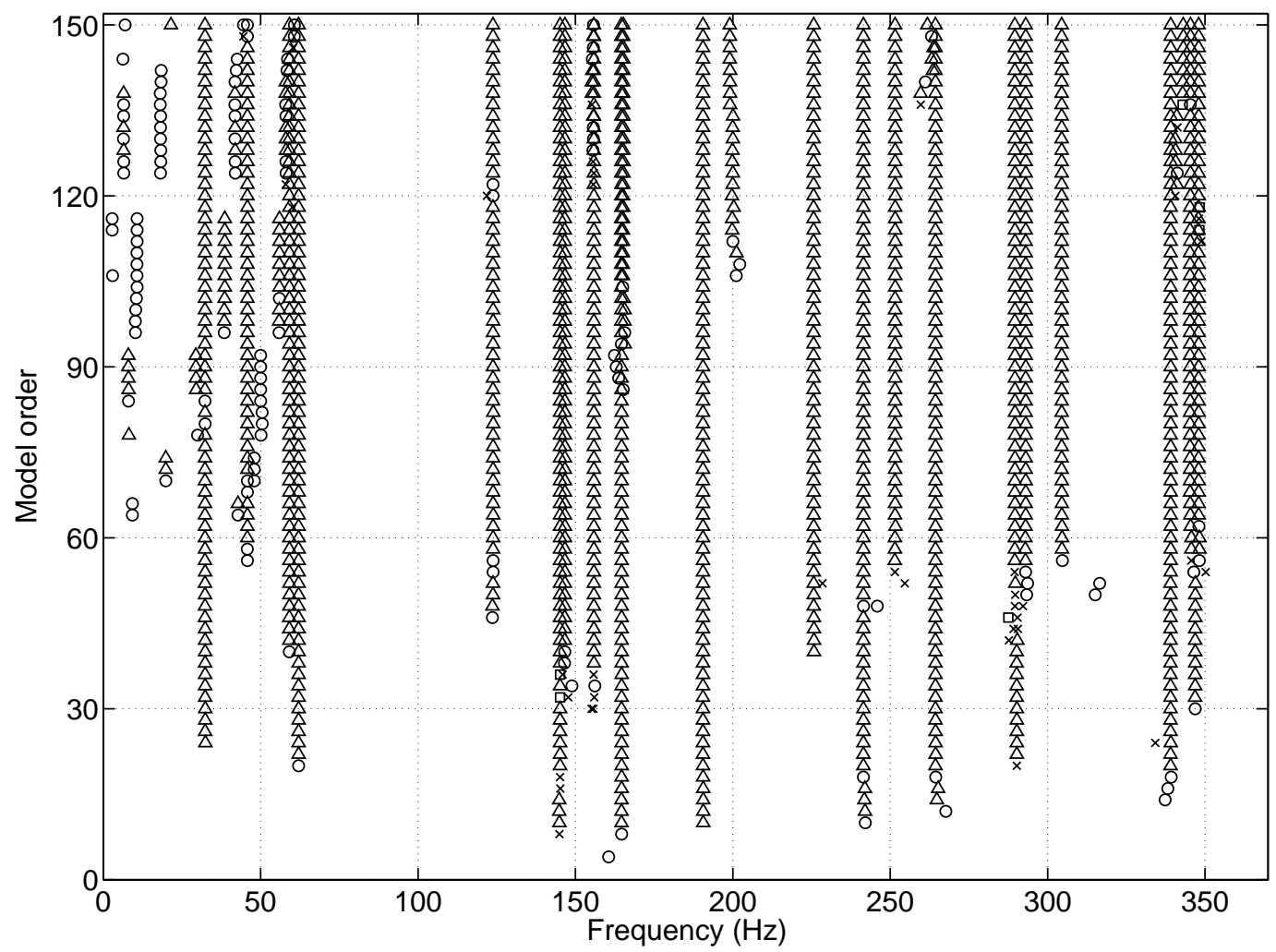

Figure 5: Stabilisation diagram computed at low level (0.16 N RMS) without noise weighting. Cross: stabilisation in natural frequency; square: extra stabilisation in damping ratio; circle: extra stabilisation in MAC; triangle: full stabilisation. 


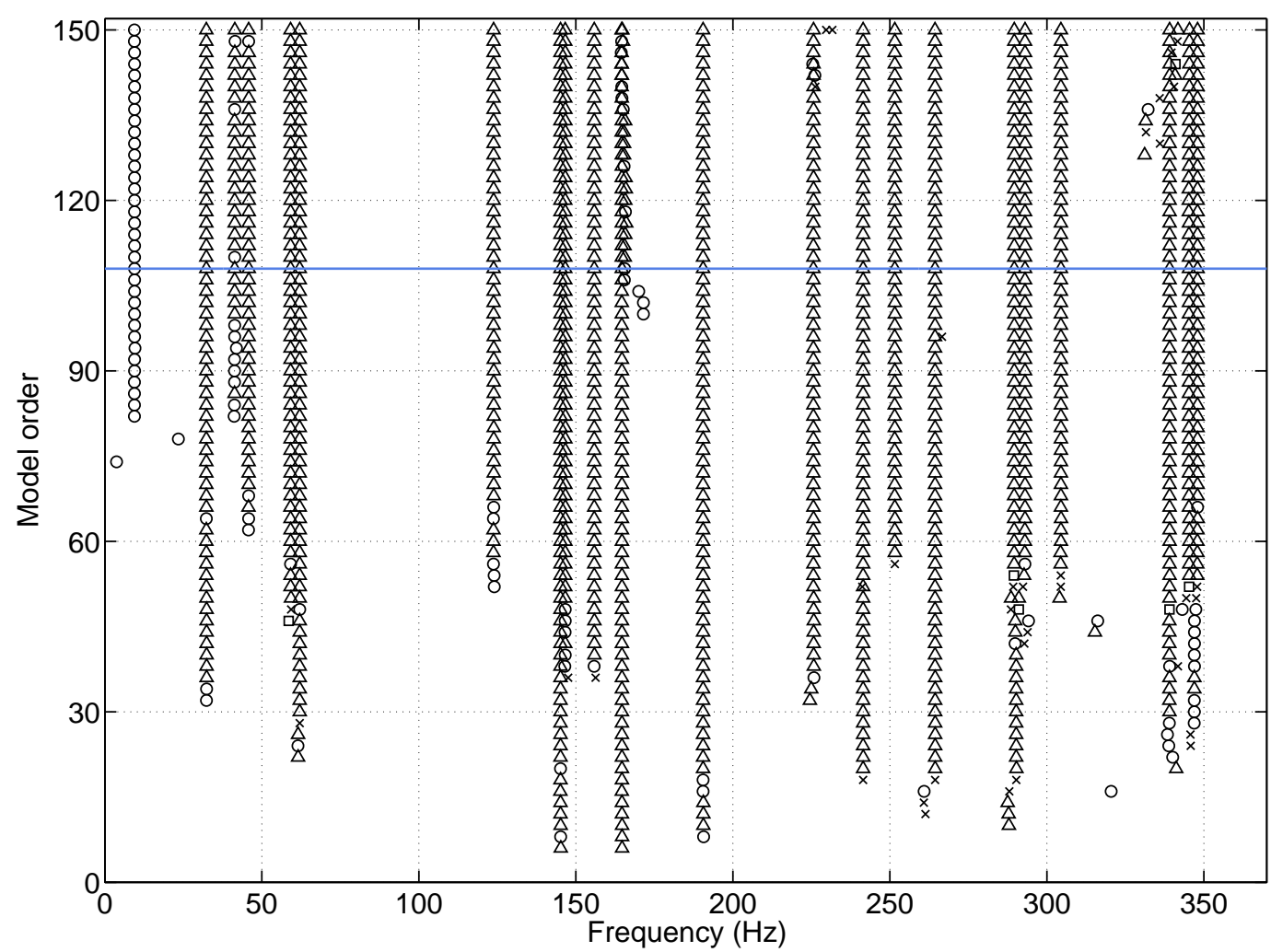

Figure 6: Stabilisation diagram computed at low level (0.16 N RMS) with noise weighting. The selected order is indicated using a blue line. Crosses, squares, circles and triangles are defined similarly to Fig. 5. 


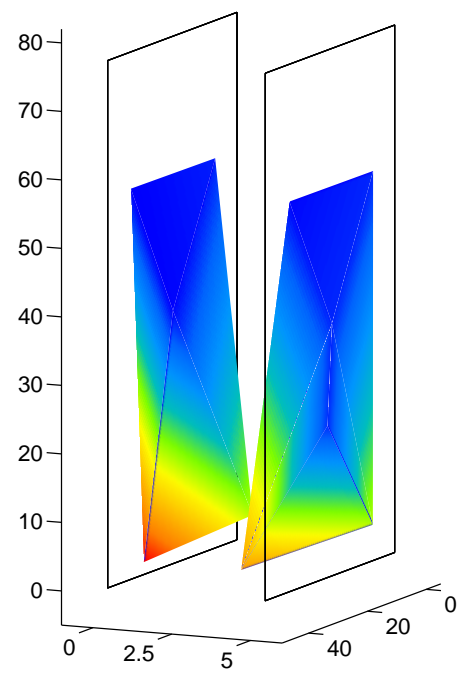

(a) Mode $1(32.32 \mathrm{~Hz})$

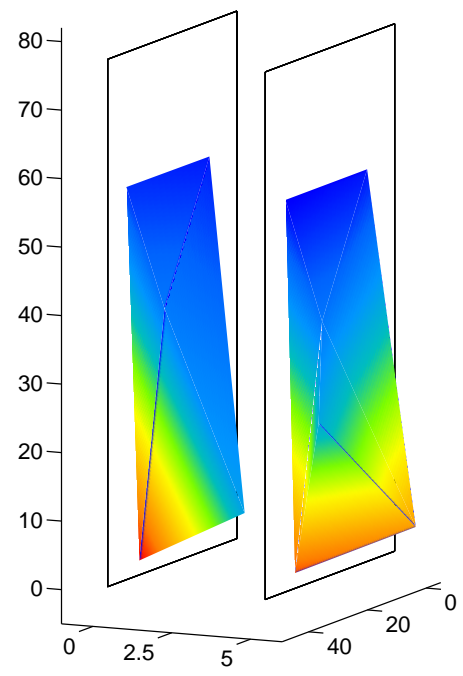

(c) Mode $7(145.13 \mathrm{~Hz})$

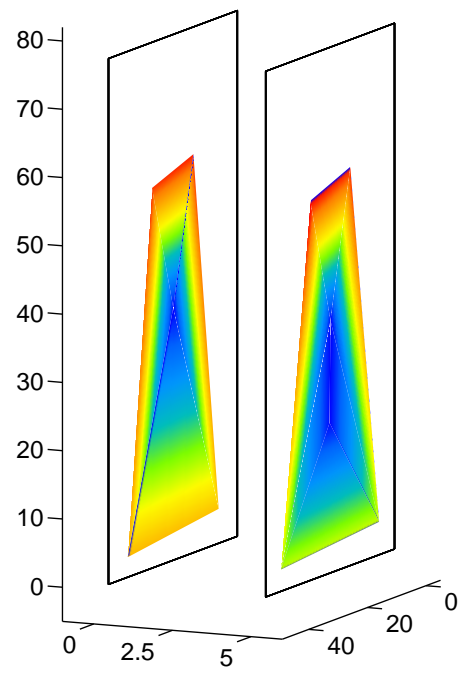

(e) Mode $12(225.68 \mathrm{~Hz})$

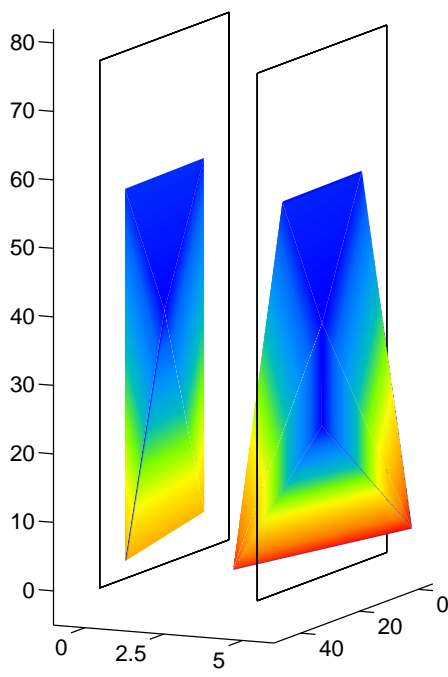

(b) Mode $3(45.83 \mathrm{~Hz})$

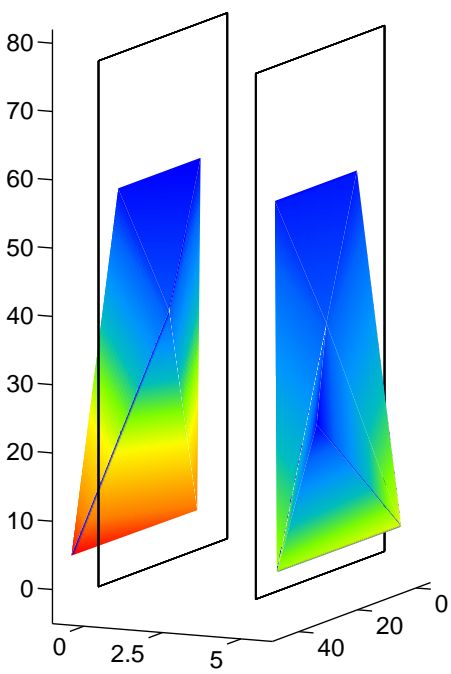

(d) Mode $11(190.52 \mathrm{~Hz})$

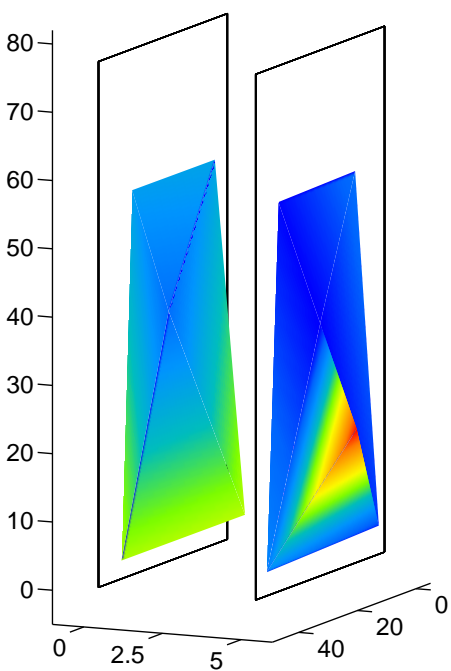

(f) Mode $15(264.37 \mathrm{~Hz})$

Figure 7: Deformed shapes corresponding to modes 1, 3, 7, 11, 12 and 15 identified at low level (0.16 $N$ RMS). Geometrical dimensions are given in centimetres. 


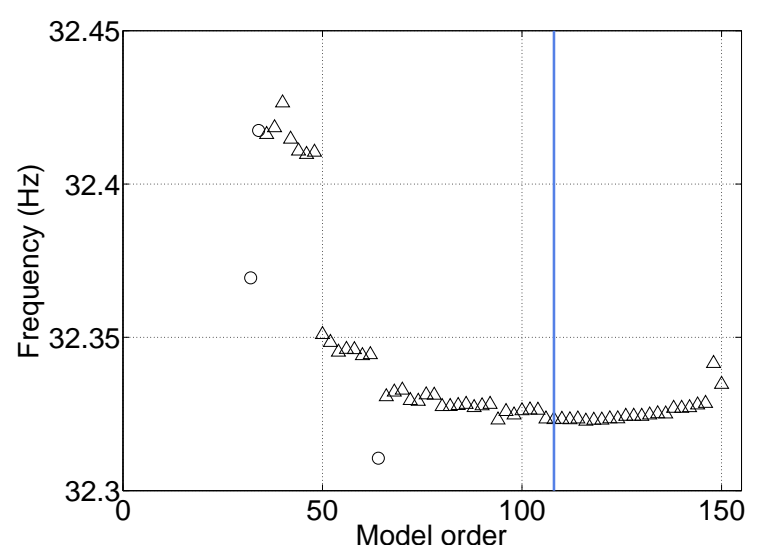

(a)

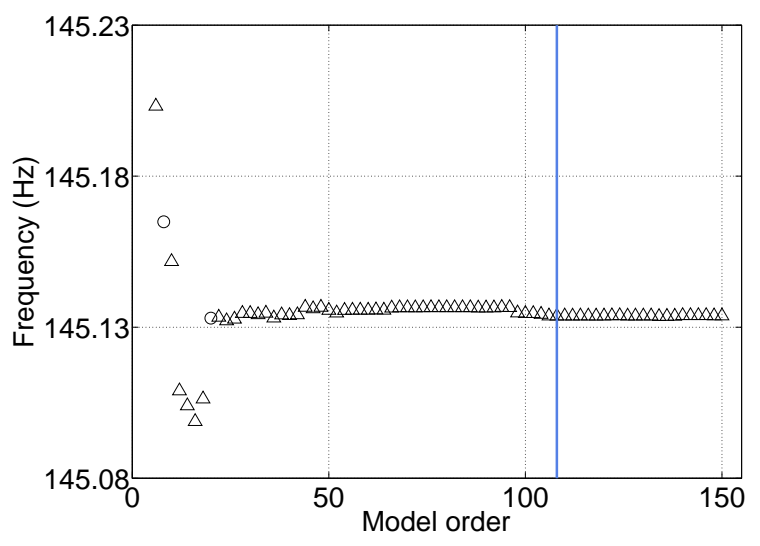

(c)

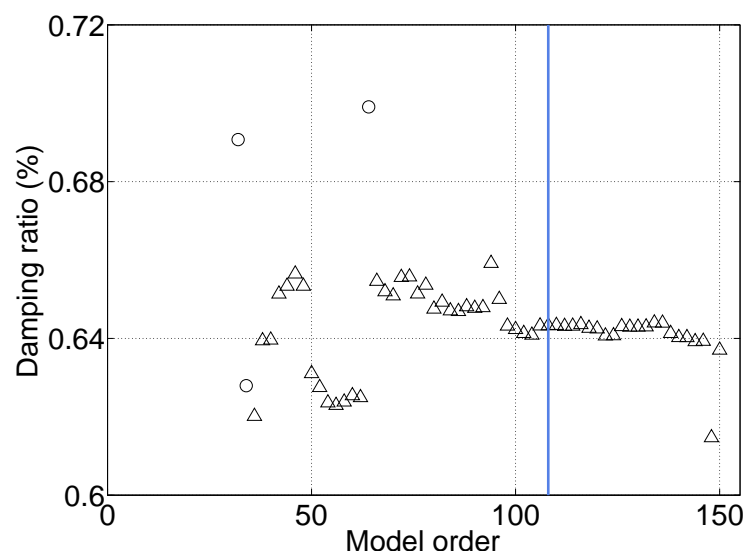

(b)

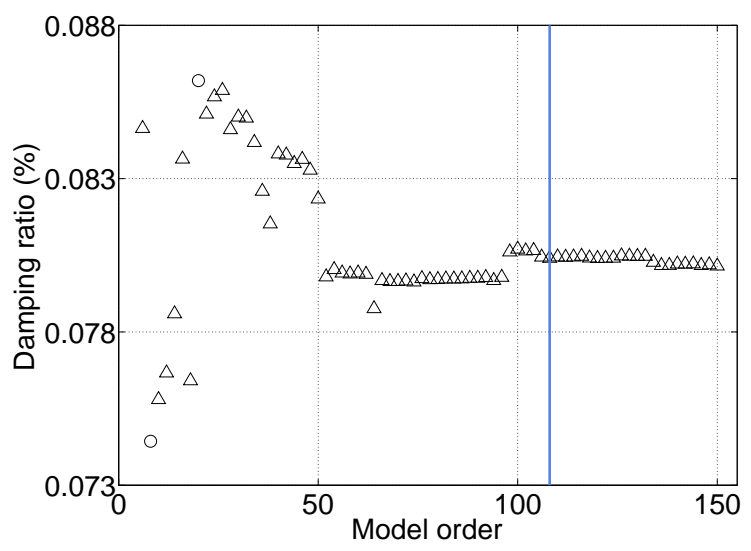

(d)

Figure 8: Frequency and damping ratio of $(\mathrm{a}-\mathrm{b})$ mode 1 and $(\mathrm{c}-\mathrm{d})$ mode 7 calculated at low level (0.16 N RMS) for increasing model orders. The selected order is indicated using blue lines. 


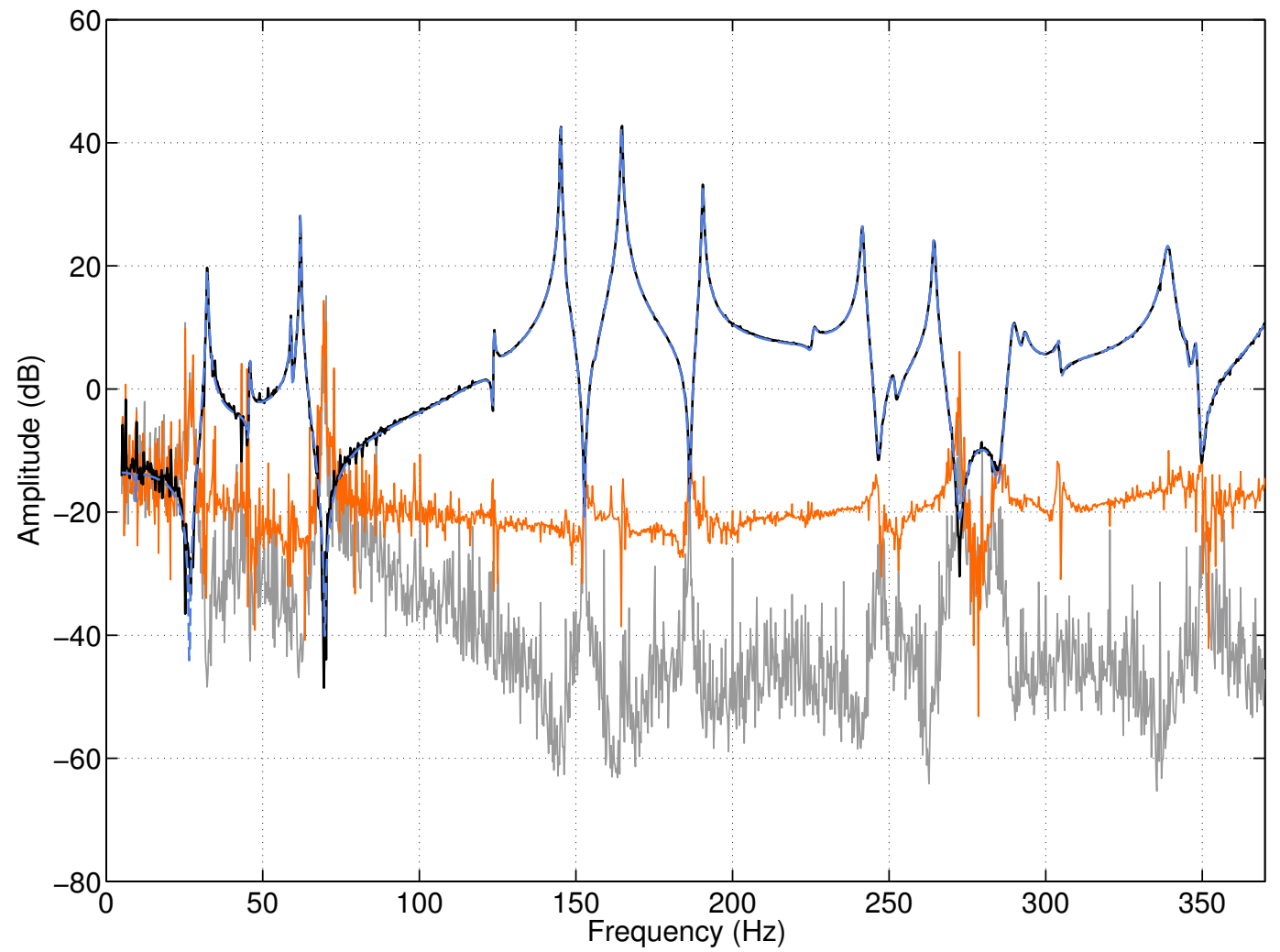

Figure 9: FRF measured at low level (0.16 $N$ RMS) at NL 4 on the front panel (in black) and corresponding FRF predicted by the linear subspace model (in dashed blue) over 5 $350 \mathrm{~Hz}$; difference between the noise variance and the measured FRF (in grey); difference between the modelling error and the measured FRF (in orange). 


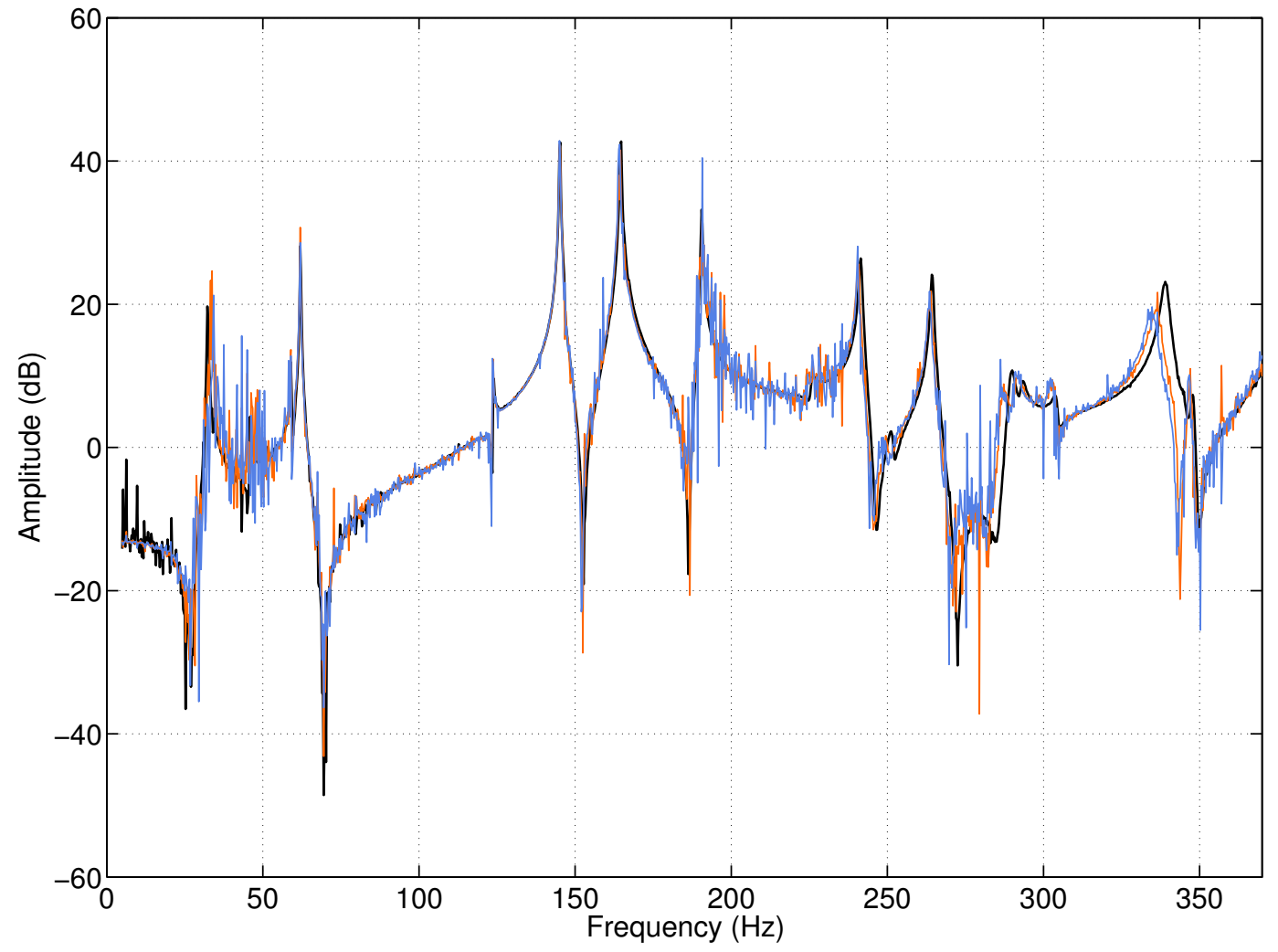

Figure 10: FRFs measured at NL 4 on the front panel over $5-350 \mathrm{~Hz}$ at $0.16 \mathrm{~N}$ (in black), $1.91 N$ (in orange) and $3.79 N$ (in blue) RMS. 


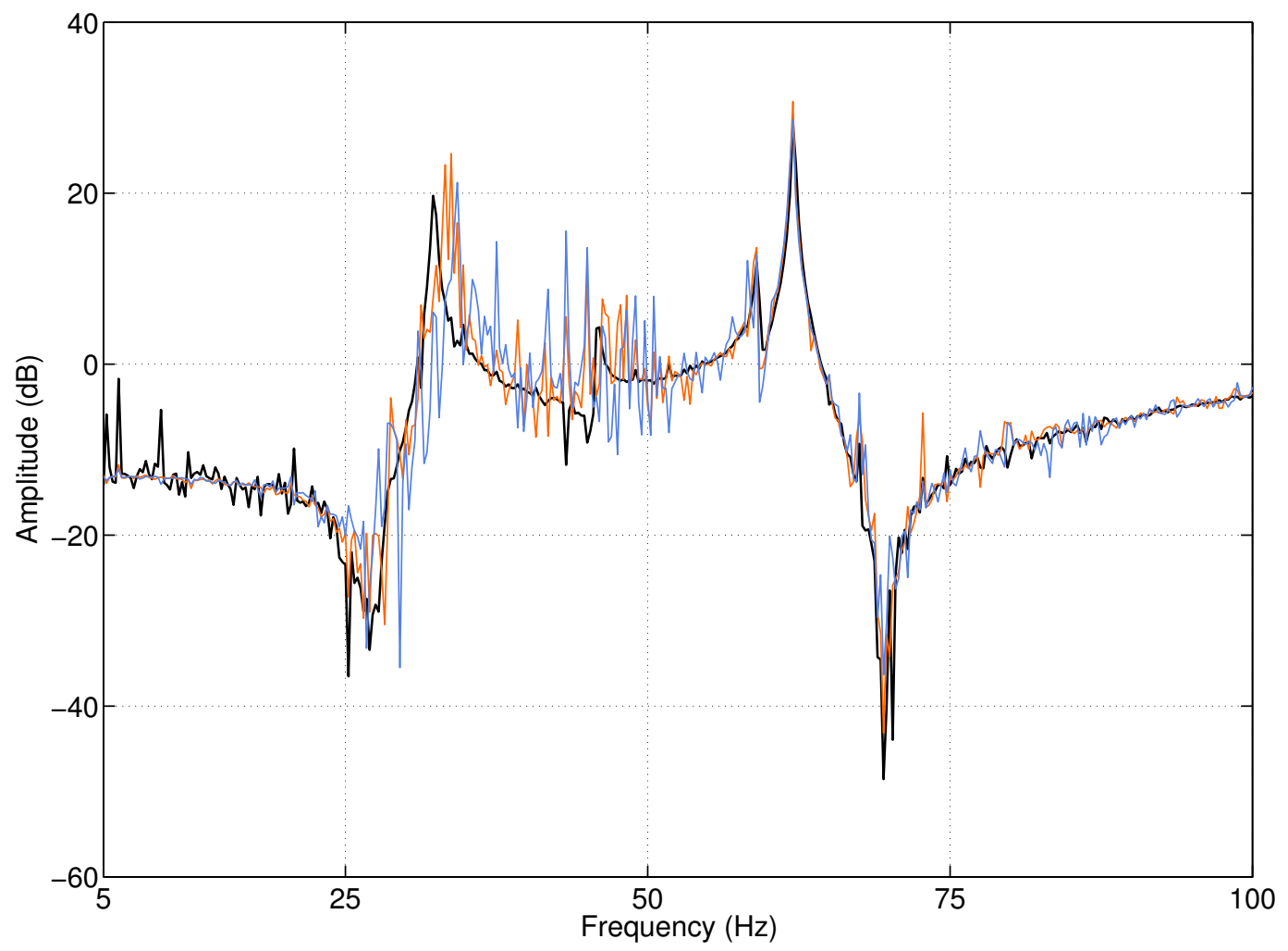

Figure 11: Close-up in the $5-100 \mathrm{~Hz}$ band of the FRFs measured at NL 4 on the front panel at $0.16 N$ (in black), $1.91 N$ (in orange) and $3.79 N$ (in blue) RMS. 


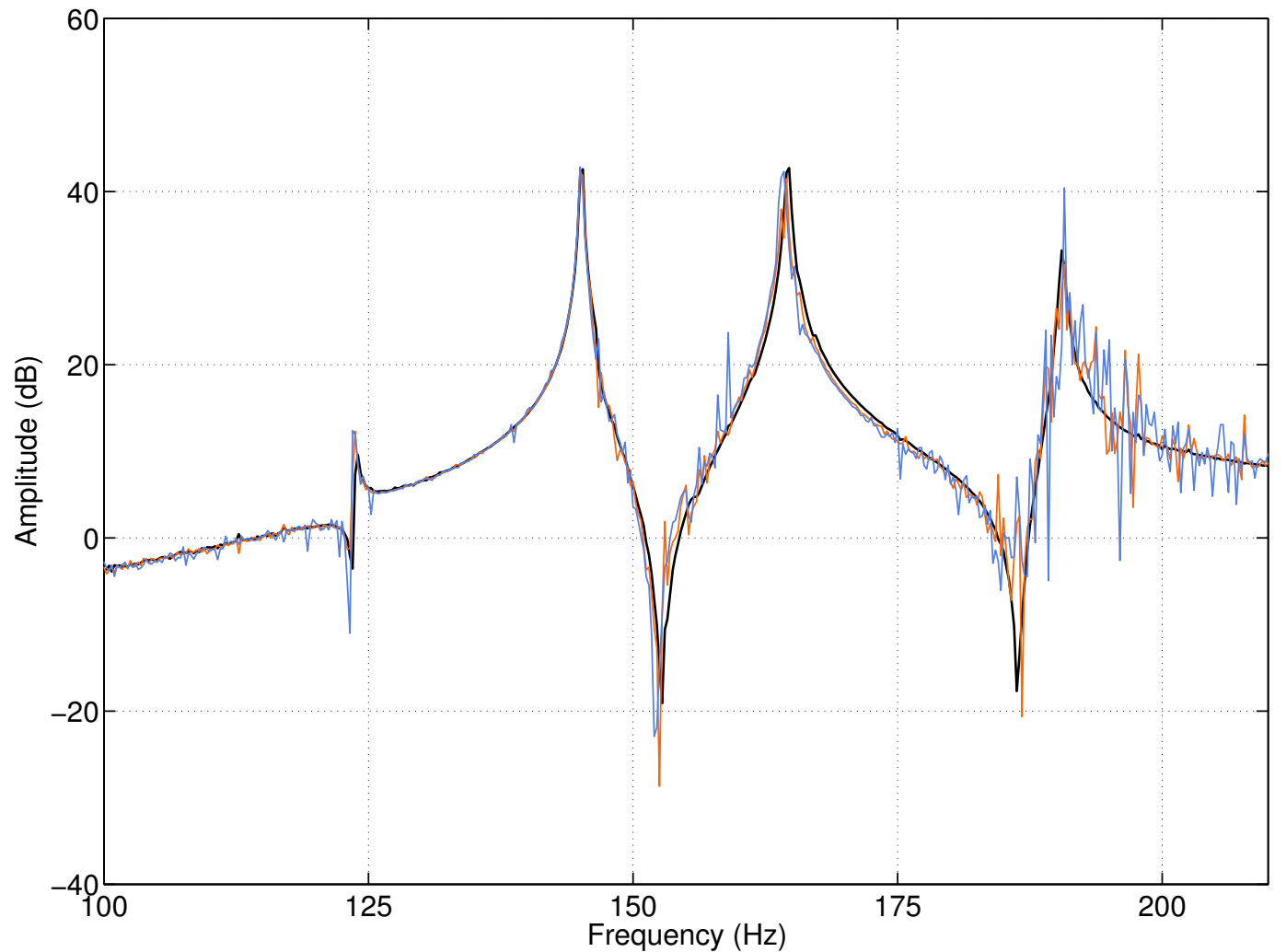

Figure 12: Close-up in the $100-200 \mathrm{~Hz}$ band of the FRFs measured at NL 4 on the front panel at $0.16 N$ (in black), $1.91 N$ (in orange) and $3.79 N$ (in blue) RMS. 


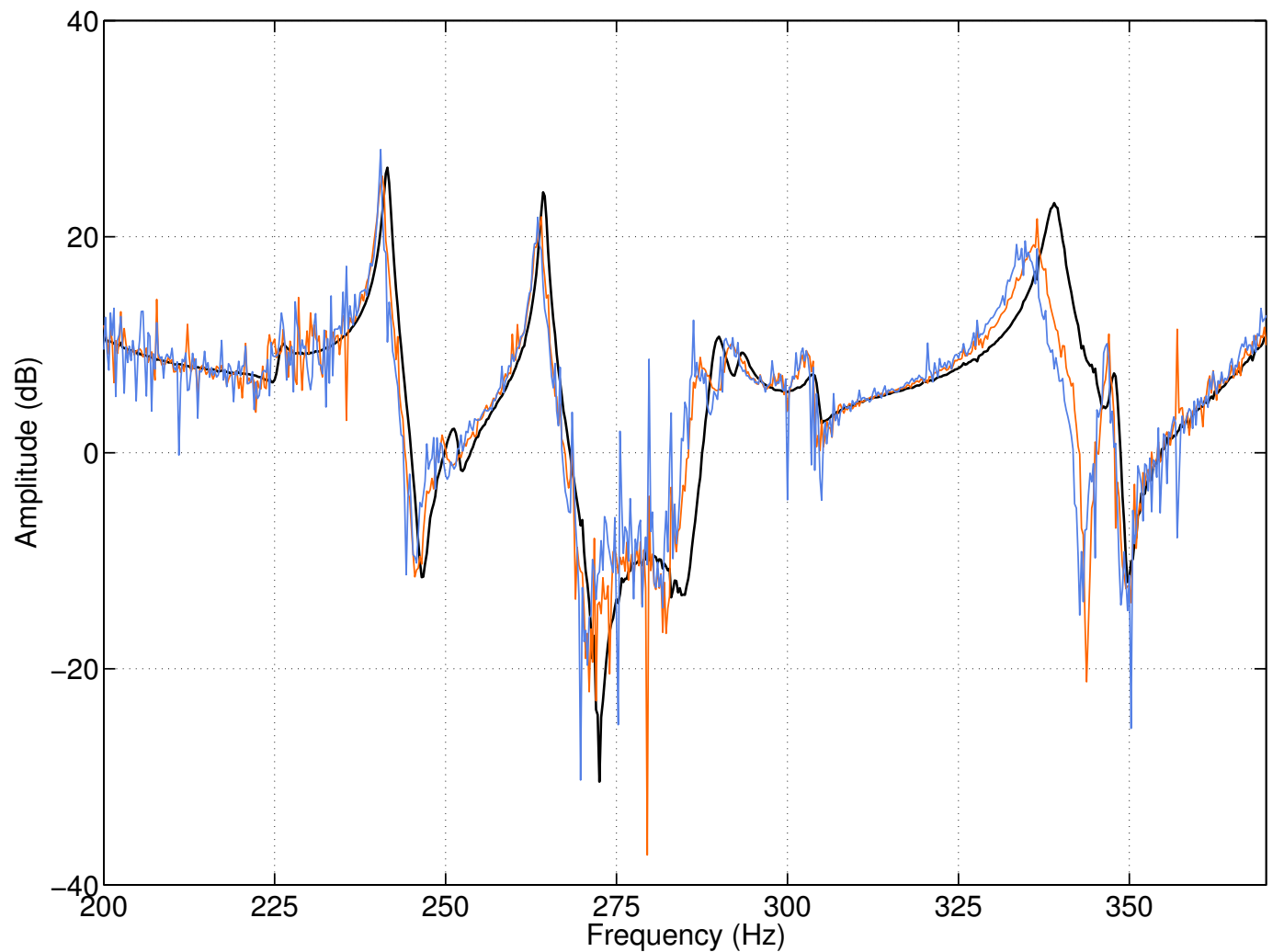

Figure 13: Close-up in the $200-350 \mathrm{~Hz}$ band of the FRFs measured at NL 4 on the front panel at $0.16 N$ (in black), $1.91 N$ (in orange) and $3.79 N$ (in blue) RMS. 


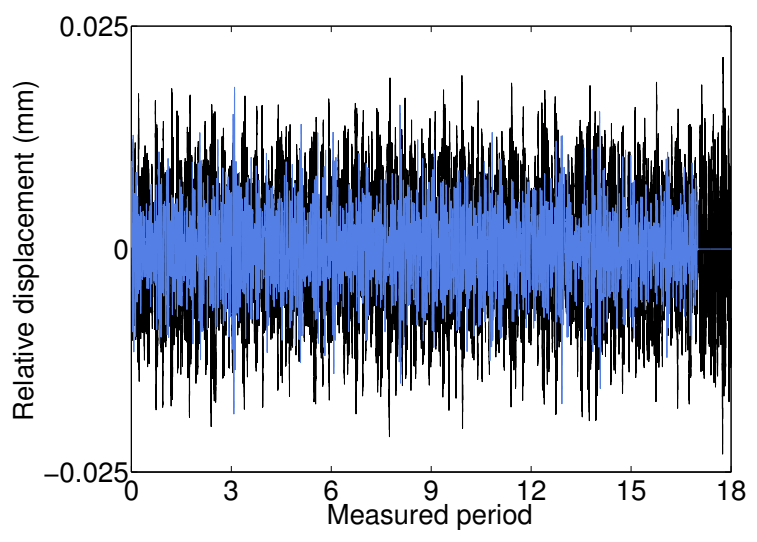

(a)

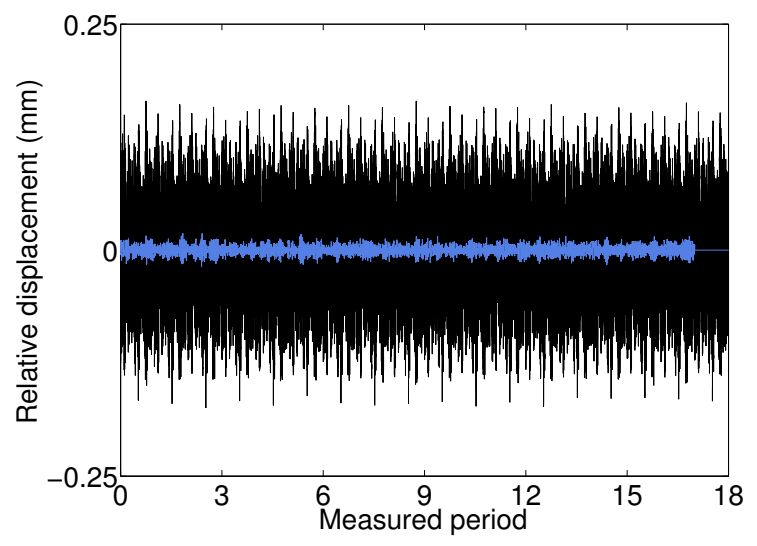

(b)

Figure 14: Relative displacement at (a) NL 1 and (b) NL 4 plotted versus time and compared to the associated noise signal. 


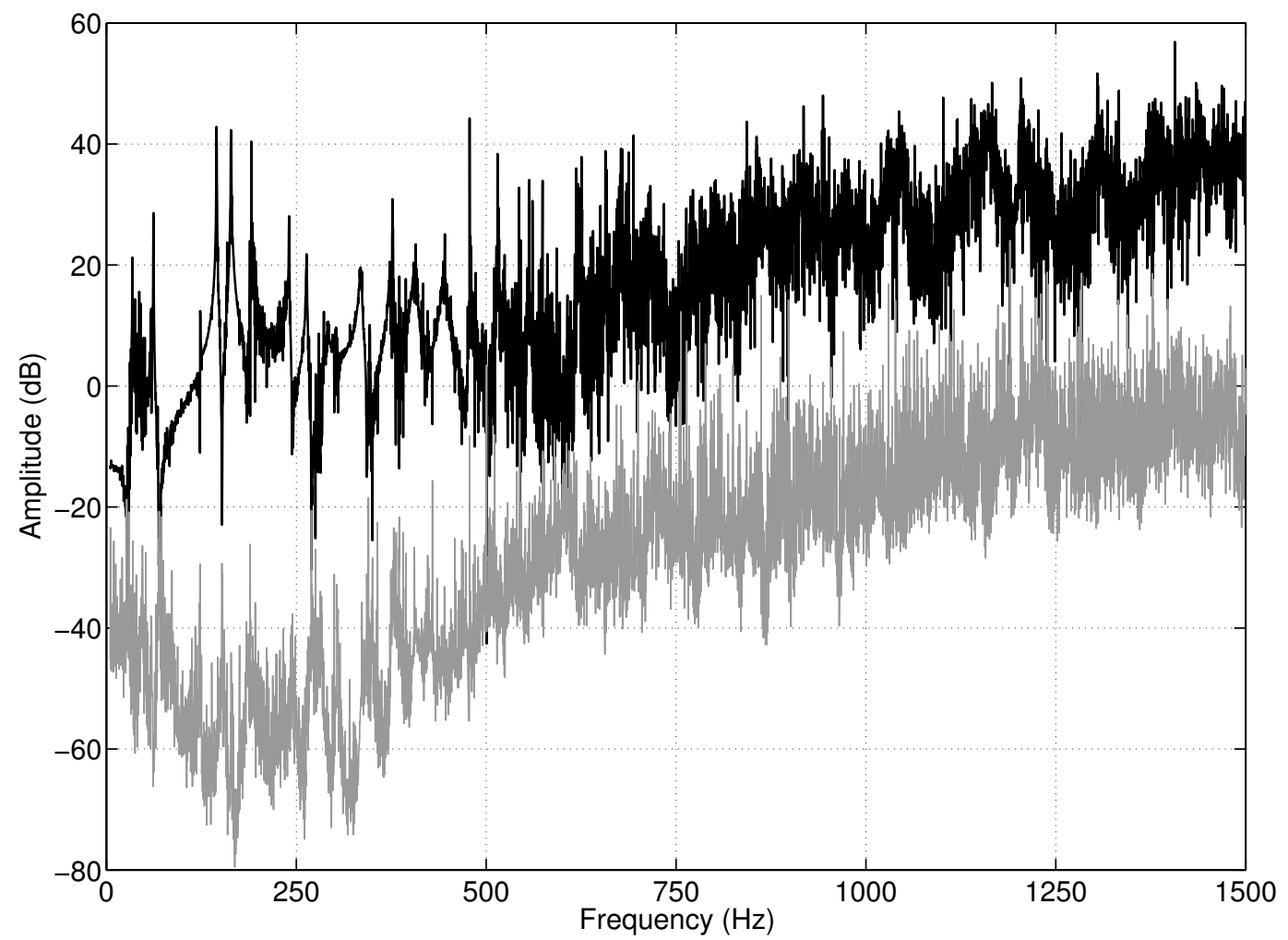

Figure 15: FRF calculated at high level (3.79 $N$ RMS) at NL 4 on the front panel over 0 - $1500 \mathrm{~Hz}$ (in black), and difference between the noise variance and the FRF (in grey). 


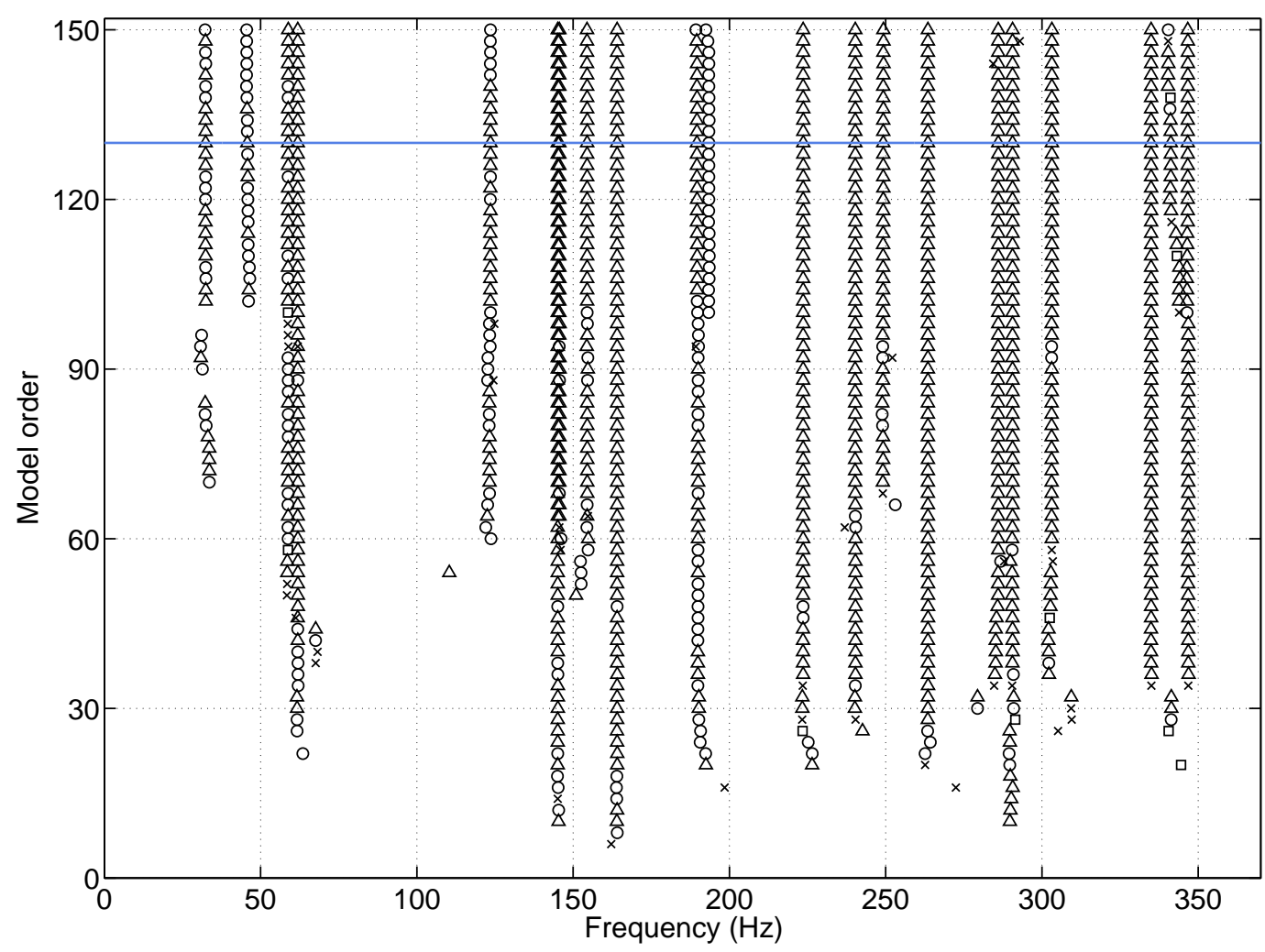

Figure 16: Stabilisation diagram computed at high level (3.79 N RMS) with noise weighting. The selected order is indicated using a blue line. Crosses, squares, circles and triangles are defined similarly to Fig. 5. 


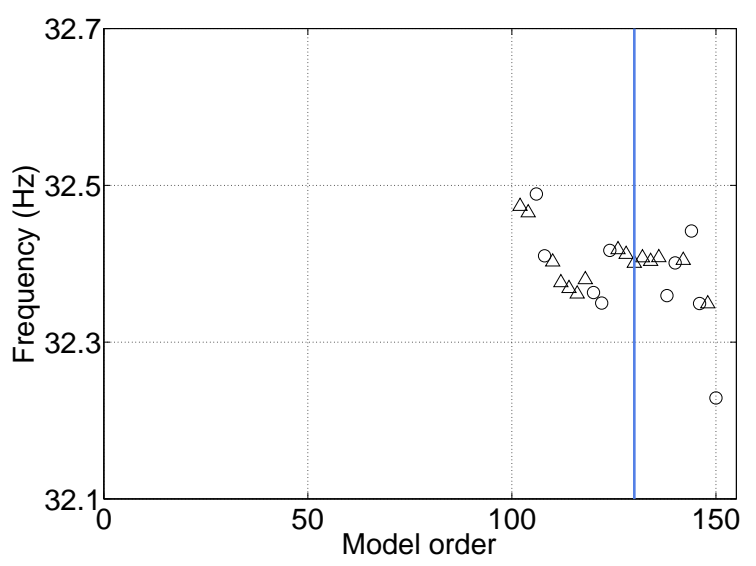

(a)

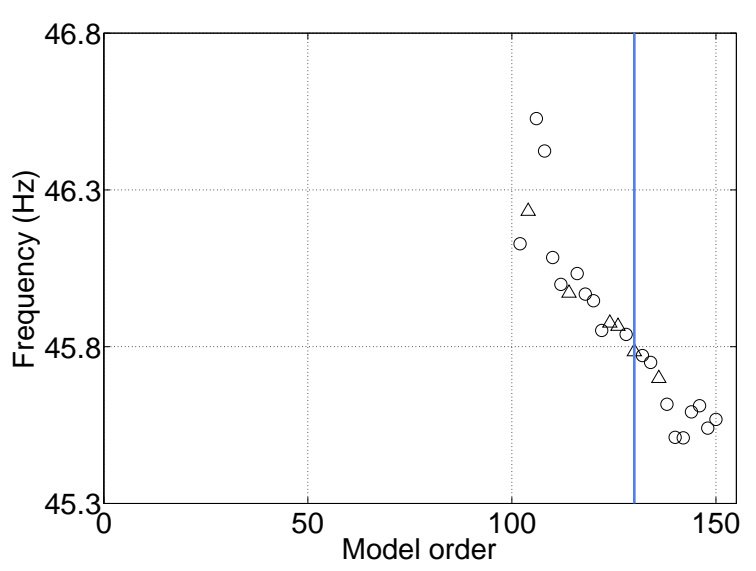

(c)

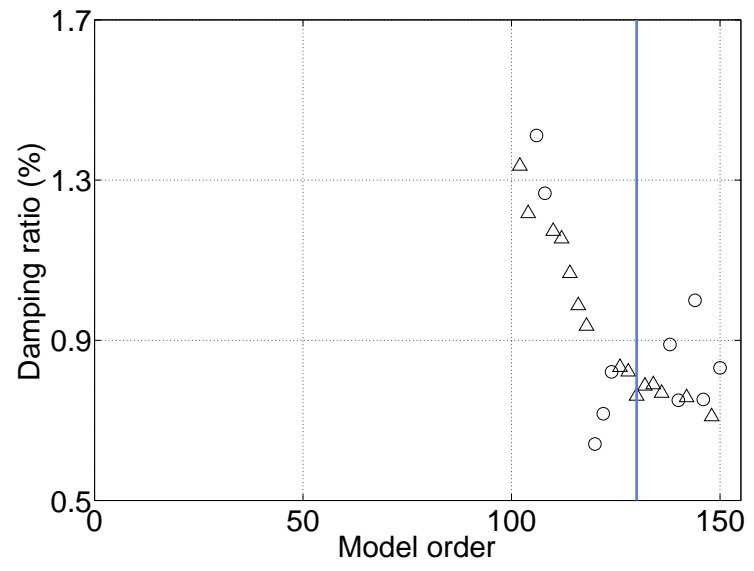

(b)

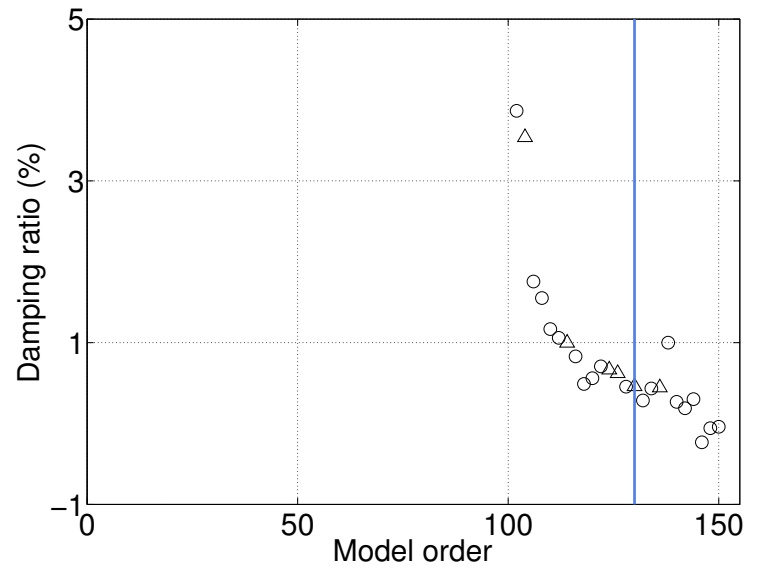

(d)

Figure 17: Linear frequency and damping ratio of modes $1(\mathrm{a}-\mathrm{b})$ and $3(\mathrm{c}-\mathrm{d})$ calculated at high level (3.79 $N$ RMS) for increasing model orders. The selected order is indicated using blue lines. 


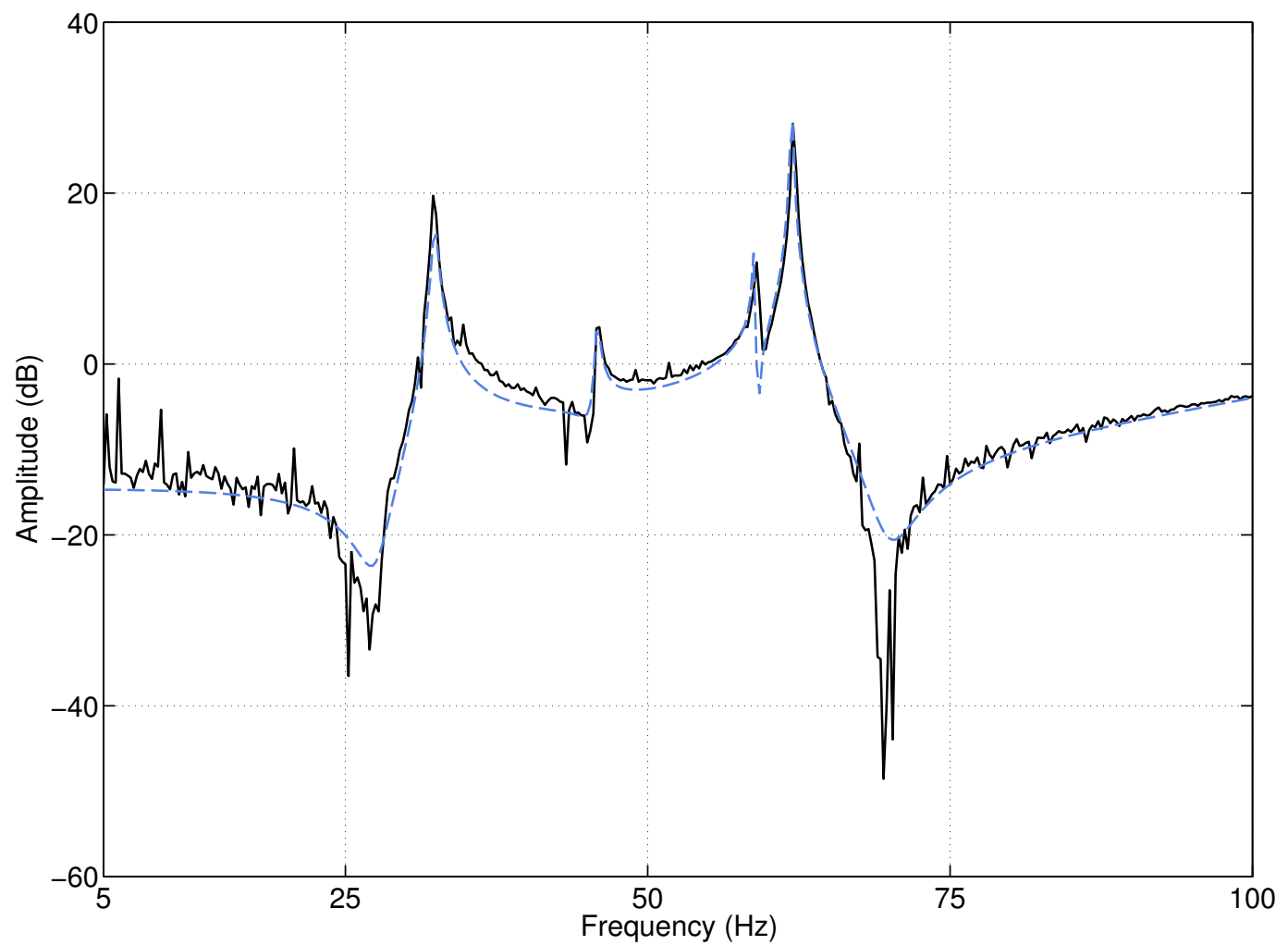

Figure 18: Comparison between the FRF measured at low level (0.16 N RMS) at NL 4 on the front panel over $5-100 \mathrm{~Hz}$ (in black), and the FRF synthesised at high level (3.79 $N$ RMS) by the FNSI algorithm (in dashed blue). 


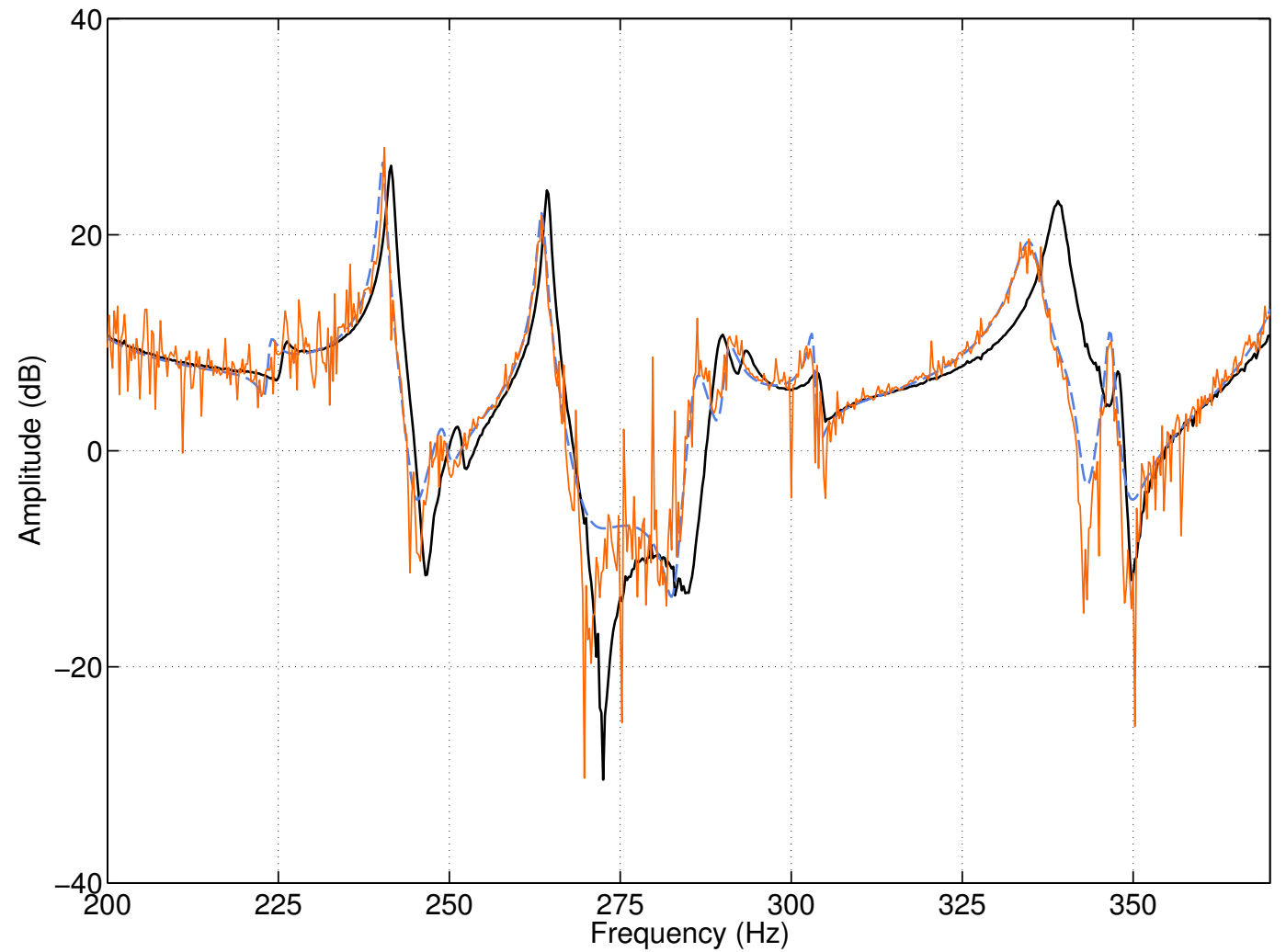

Figure 19: Comparison between the FRF measured at low level (0.16 $N$ RMS) at NL 4 on the front panel over $200-350 \mathrm{~Hz}$ (in black), and the FRF reconstructed at high level (3.79 N RMS) by the FNSI algorithm (in dashed blue); the FRF measured at high level (3.79 $N$ RMS) is also plotted (in orange). 


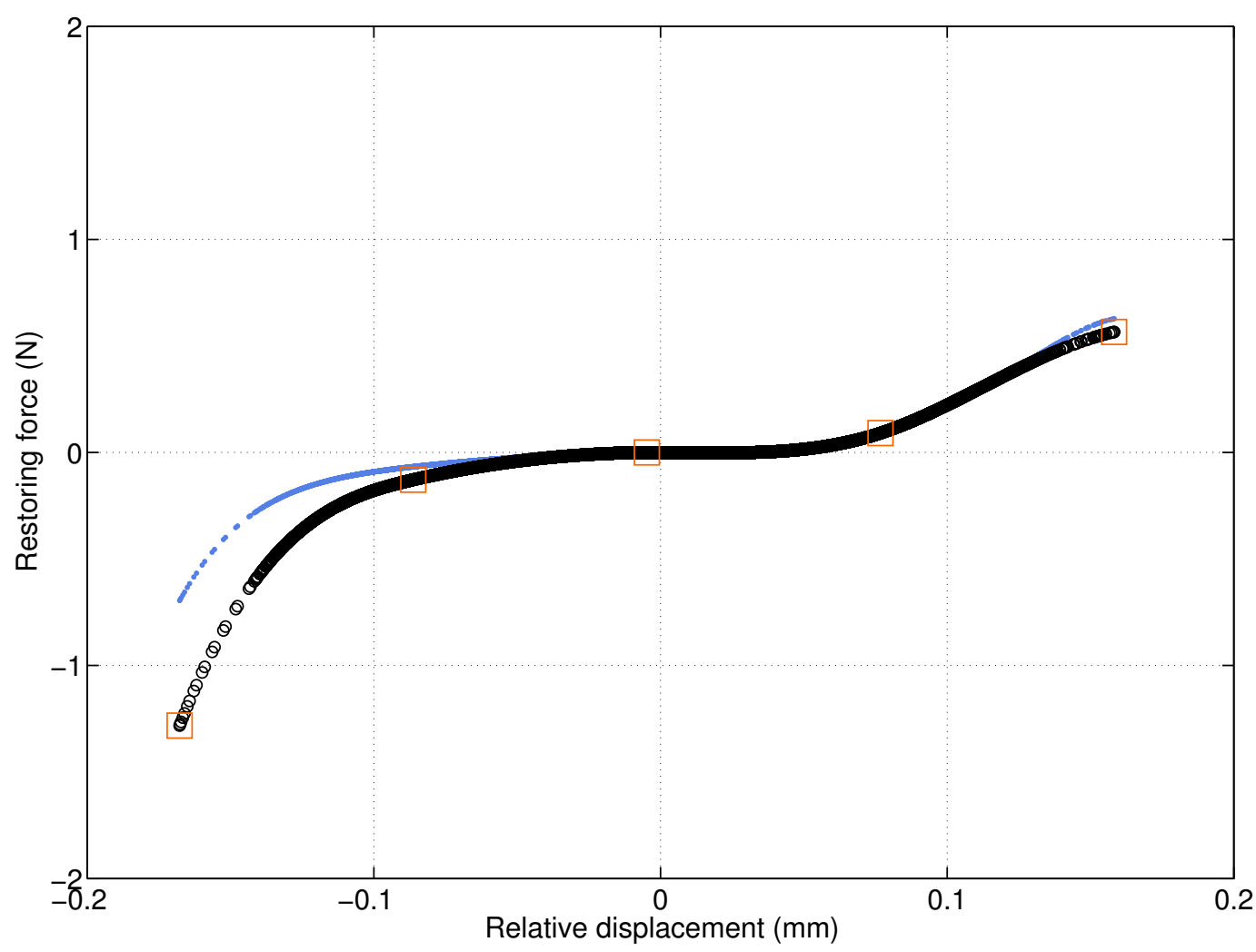

(a)

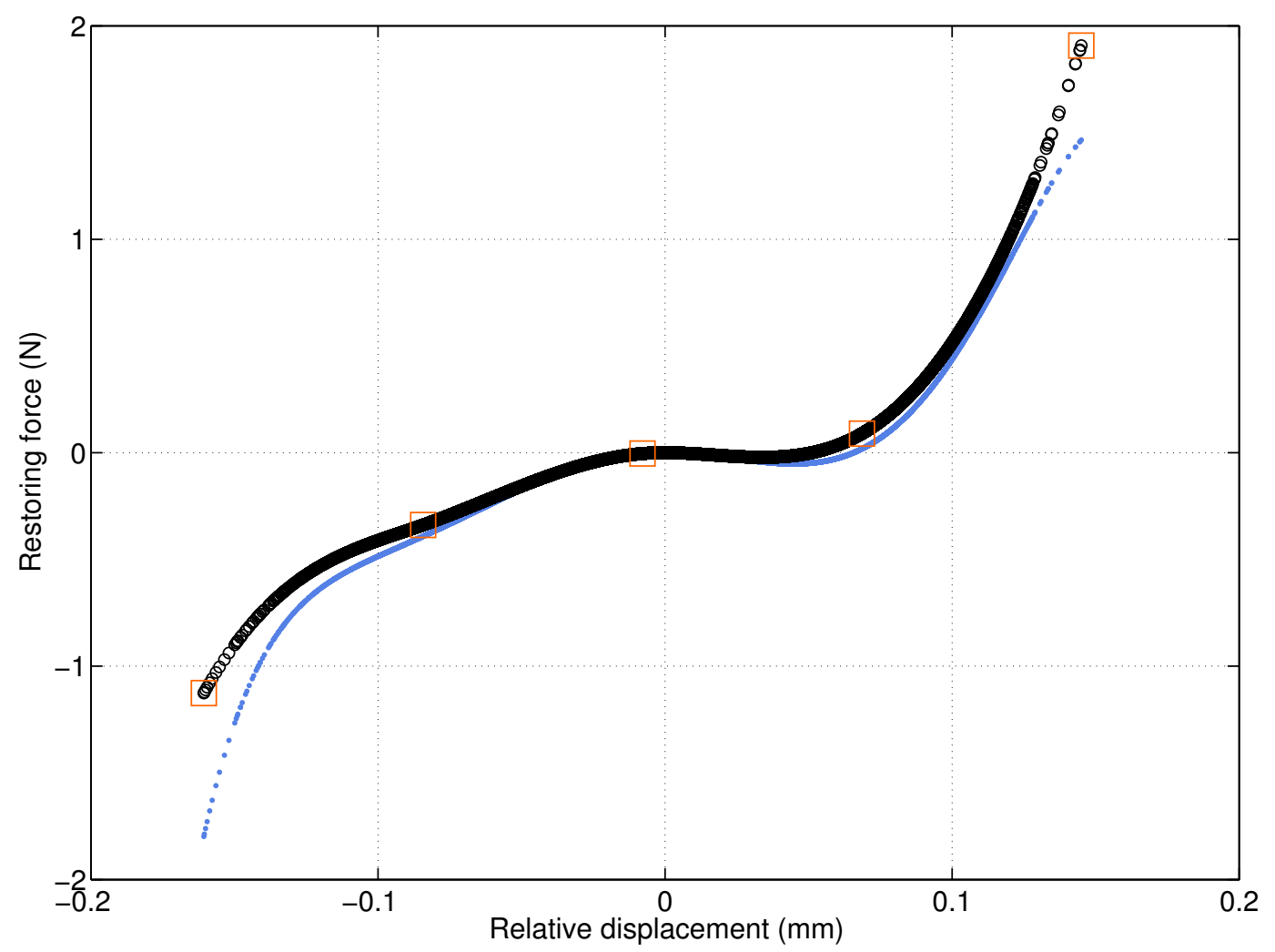

(b)

Figure 20: Nonlinear stiffness curve constructed at high level (3.79 N RMS) using cubic splines (black circles) and sixth-order polynomials (blue dots): (a) NL 4; (b) NL 5. Orange squares localise the 5 chosen knots of the splines. 


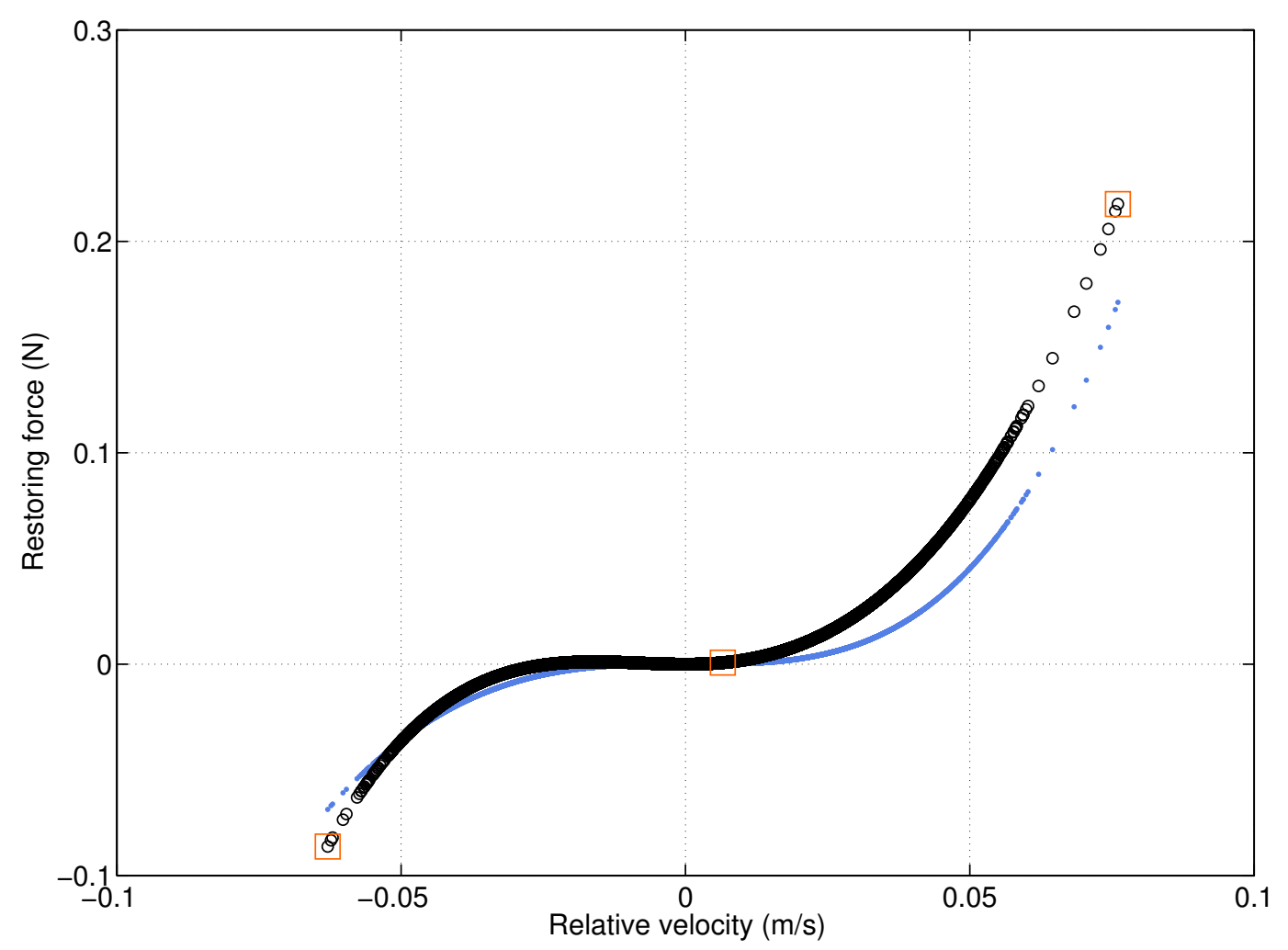

(a)

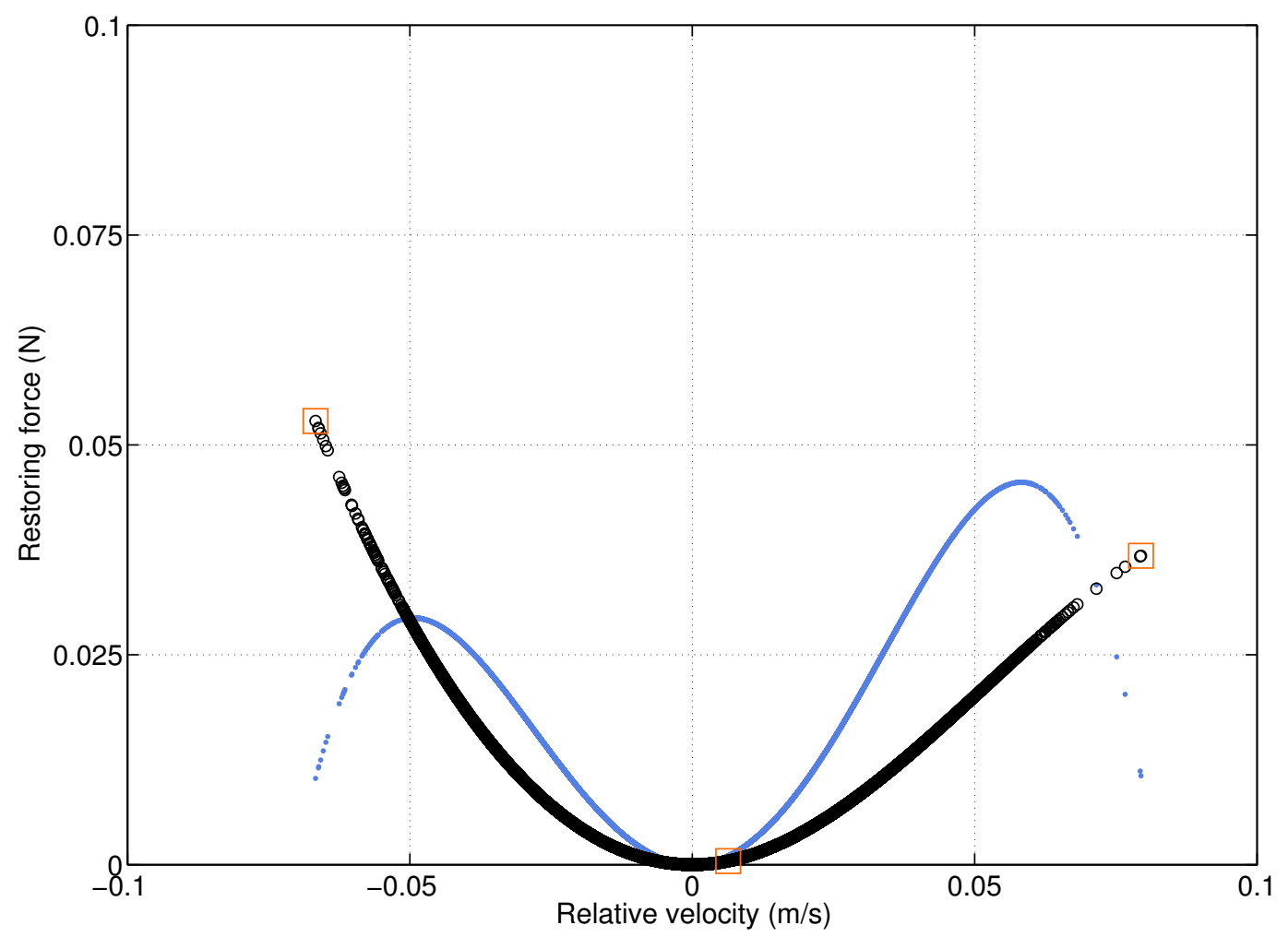

(b)

Figure 21: Nonlinear damping curve constructed at high level (3.79 N RMS) using cubic splines (black circles) and fourth-order polynomials (blue dots): (a) NL 4; (b) NL 5. Orange squares localise the 3 chosen knots of the splines. 


\section{List of Tables}

1 Natural frequencies and damping ratios of the 21 modes identified at lowlevel $(0.16 \mathrm{~N}$ RMS $)$ in the $5-350 \mathrm{~Hz}$ band. . . . . . . . . . . . . . . . 45

2 Natural frequencies $(\mathrm{Hz})$ of a selection of modes estimated at low level (second column), using a spline model at high level (third column), using a linear model at high level (fourth column) and using a polynomial model with the same number of parameter as the spline model at high level (fifth column). . . . . . . . . . . . . . . . . . 46

3 Damping ratios (\%) of a selection of modes estimated at low level (second column), using a spline model at high level (third column) and using a polynomial model with the same number of parameter as the spline model at high level (fourth column). . . . . . . . . . . . . . . . . . . . . 47 


\begin{tabular}{ccc}
\hline Mode & Natural frequency $(H z)$ & Damping ratio $(\%)$ \\
1 & 32.32 & 0.64 \\
2 & 41.36 & 0.80 \\
3 & 45.83 & 0.31 \\
4 & 59.14 & 0.27 \\
5 & 62.07 & 0.16 \\
6 & 123.82 & 0.14 \\
7 & 145.13 & 0.08 \\
8 & 146.64 & 0.22 \\
9 & 155.87 & 0.39 \\
10 & 164.62 & 0.14 \\
11 & 190.52 & 0.14 \\
12 & 225.68 & 0.32 \\
13 & 241.50 & 0.20 \\
14 & 251.56 & 0.36 \\
15 & 264.37 & 0.17 \\
17 & 289.60 & 0.43 \\
18 & 293.05 & 0.31 \\
19 & 304.48 & 0.15 \\
20 & 339.08 & 0.36 \\
21 & 345.24 & 0.38 \\
\hline
\end{tabular}

Table 1: Natural frequencies and damping ratios of the 21 modes identified at low-level (0.16 $\mathrm{N}$ RMS) in the $5-350 \mathrm{~Hz}$ band. 


\begin{tabular}{ccccc}
\hline Mode & $\begin{array}{c}\text { Linear model } \\
\text { at low level }\end{array}$ & $\begin{array}{c}\text { Spline model } \\
\text { at high level }\end{array}$ & $\begin{array}{c}\text { Linear model } \\
\text { at high level }\end{array}$ & $\begin{array}{c}\text { Polynomial model } \\
\text { at high level }\end{array}$ \\
1 & 32.32 & 32.39 & 34.12 & 31.98 \\
3 & 45.83 & 45.80 & 48.53 & 46.21 \\
7 & 145.13 & 145.08 & 145.09 & 145.08 \\
10 & 164.62 & 164.07 & 164.15 & 164.07 \\
11 & 190.52 & 189.64 & 190.86 & 189.88 \\
15 & 264.37 & 263.53 & 263.52 & 263.53 \\
16 & 289.60 & 286.04 & 286.09 & 286.01 \\
19 & 339.08 & 334.95 & 334.97 & 334.95 \\
\hline
\end{tabular}

Table 2: Natural frequencies $(H z)$ of a selection of modes estimated at low level (second column), using a spline model at high level (third column), using a linear model at high level (fourth column) and using a polynomial model with the same number of parameter as the spline model at high level (fifth column). 


\begin{tabular}{cccc}
\hline Mode & $\begin{array}{c}\text { Linear model } \\
\text { at low level }\end{array}$ & $\begin{array}{c}\text { Spline model } \\
\text { at high level }\end{array}$ & $\begin{array}{c}\text { Polynomial model } \\
\text { at high level }\end{array}$ \\
1 & 0.64 & 0.77 & 0.83 \\
3 & 0.31 & 0.49 & 1.29 \\
7 & 0.08 & 0.09 & 0.09 \\
10 & 0.14 & 0.14 & 0.15 \\
11 & 0.14 & 0.37 & 0.46 \\
15 & 0.17 & 0.21 & 0.21 \\
16 & 0.43 & 0.46 & 0.46 \\
19 & 0.36 & 0.53 & 0.54 \\
\hline
\end{tabular}

Table 3: Damping ratios (\%) of a selection of modes estimated at low level (second column), using a spline model at high level (third column) and using a polynomial model with the same number of parameter as the spline model at high level (fourth column). 